\title{
ABSTRACTS \\ SASOP Biological Psychiatry Congress 2013
}

\author{
29 August - 1 September 2013 \\ Wild Coast Sun, Port Edward, South Africa
}

S Afr J Psych 2013;19(3):91-126. DOI:10.7196/SAJP.473

\section{ORAL PRESENTATIONS}

Bipolar disorder not otherwise specified - overdiagnosed or underdiagnosed?

\section{E Allers}

Psychiatrist in Private Practice

kopshop@global.co.za

The total cost spend on beneficiaries of medical schemes with the diagnosis of bipolar disorder has escalated by nearly $30 \%$ annually over the past 5 years. The risk of managing the cost of this group of patients for the medical schemes has placed them on the schemes' priority list. General practitioners have been commenting on the sudden rise in the diagnosis of bipolar disorder and have queried psychiatrists about this apparent overdiagnosis. The awareness of the diagnosis of bipolar disorder has highlighted it among the public and psychiatrists. Several articles comment on the underdiagnosis as the overdiagnosis of bipolar disorder. This presentation aims to analyse the data from South Africa as well as published international data to put the debate in perspective.

The prognosis of major depression untreated and treated: Does the data reflect the true picture of the prognosis of this very common disorder?

E Allers

Psychiatrist in Private Practice

kopshop@global.co.za

Major depression is currently the most common psychiatric disorder and represents one of the disorders with the worst prognosis regarding functioning. A scrutiny of the evidence suggests the overall prognosis of treated patients to be only marginally better than placebo. This presentation aims to analyse the data of short-, medium- and long-term studies closely to determine the true prognosis of major depression of treated and untreated patients.

Can we prolong our patients' life expectancy? Providing a better quality of life for patients with severe mental illness O A Betancourt

Department of Psychiatry, Faculty of Health Sciences,

Walter Sisulu University, Mthatha, South Africa

orlando@intekom.co.za
People with severe mental illness (SMI) die 25 years earlier than members of the general population. This excess mortality is associated with physical illness such as nutritional and metabolic diseases, cardiovascular, viral and respiratory tract diseases and possible obesity-related cancer, among other causes. Statistically, $4.9 \%$ of patients suffering from schizophrenia die of suicide usually near the onset of the illness. Cigarette smoking is highly prevalent (50 - 80\%) among patients with SMI, with all its consequences. In a South African study, HIV sero-prevalence among those with SMI was higher than that of the general population. Is well known that some atypical antipsychotics promote weight gain and increase insulin resistance - in general, antipsychotic drugs cause, predispose and/ or aggravate medical conditions. However, the good news is that this increase in morbidity and mortality is mainly due to preventable conditions. The question is: can we prolong our patients' lives? Can we make their life better? The answer is yes: by targeting modifiable risk factors (obesity, smoking, physical inactivity, drug abuse and bad diet habits), prescribing appropriate medications that can minimise symptoms, increase adherence and allow people with SMI to function as independently as possible. Patients differ in how they respond to different antipsychotics. Pharmacotherapy for patients with SMI must be tailored to individuals. The routine in our clinics should include wellness, lifestyle and quality of life as part of the process and final result of management of SMI. The full mental health team has to be involved in the integral management. Psycho-education must be given to patients, families and community members to make them part of the programme. All these can be done in South Africa at present.

\section{The scope of ECT practice in South Africa LBenson-Martin, ${ }^{\star}$ P Milligan \\ University of Cape Town and Valkenberg Hospital, Cape Town, South Africa \\ *j.benson-martin@uct.ac.za}

Background. Electroconvulsive therapy (ECT) involves the administration of an electrical current to the brain in order to producing a tonic-clonic seizure. ECT is an effective and safe procedure used in the treatment of severe mental illnesses such as major depression, mania and schizophrenia. Studies from the Middle East, Asia and Europe show varying techniques and utilisation rates of ECT practice. Resource-rich countries tend to use this practice less 
and in a more regulated fashion, while developing countries perform ECT more frequently and often unmodified.

Objectives. Currently, little is known about the characteristics of ECT practice in South Africa. This study aims to determine current ECT practice in South Africa and to compare it with reported ECT practice in other parts of the world.

Methods. This is a descriptive, analytical study describing the characteristics of ECT practice in South Africa. The study population consisted of doctors and nurses who practiced ECT in 2011/2012. Both private and state facilities were included. Initially contact was made with all hospitals with mental health facilities to ascertain whether an ECT machine was present on site. Once formal approval was obtained from the appropriate designated bodies, questionnaires were sent to clinical staff involved in ECT at active sites. The 36-item questionnaire covered relevant questions on: utilisation rates, equipment, staffing, practice and monitoring parameters, and main indications for use.

Results. Forty-two institutions had an ECT machine on site, of which 13 institutions reported non-use. Questionnaires were sent to 29 active ECT sites: $83 \%(n=24)$ of facilities responded to the questionnaire, of which $72 \%(n=21)$ responded to the ECT utilisation questions. The number of ECT procedures/10 000 population per year (ppy) is 1.19, while the number of persons treated with ECT/10 000 ppy is 0.22 . Modified ECT is performed in 6 of the 9 provinces by psychiatrists, registrars or medical officers. The vast majority of patients are between the ages of 18 and 59. The most common indication for ECT is depression.

Conclusion. The utilisation rate in South Africa is similar to that of countries such as Bulgaria, Poland and Hong Kong but less than that of some western countries. ECT practices in South Africa generally follow international guidelines.

\section{Biomarkers for schizophrenia: Can we evolve like cancer therapeutics? \\ P Buckley \\ Medical College of Georgia, Georgia Regents University, \\ Augusta, Georgia, USA \\ pbuckley@gru.edu}

Rapidly expanding knowledge in neuroscience, along with new developments in technology and broadening domains of outcome, collectively create new opportunities for advancing disease detection and developing more effective treatments for schizophrenia. These paths of innovation in neuroscience call for a more rapid translation of basic and clinical research findings into clinical practice. The concerted efforts to develop biomarkers for psychiatry in general, and specifically for psychosis, are consistent with this direction and seek to leverage advances of emerging importance in schizophrenia. Identification of biomarkers in schizophrenia is still in the early stages of development compared with other areas of medicine, most notably cancer therapeutics. This presentation will describe the role of biomarkers, with a focus on brain-derived neurotrophic factor, and the implications thereof for predicting response to treatments in schizophrenia.

\author{
Relapse in schizophrenia: Major challenges in \\ prediction and prevention \\ P Buckley \\ Medical College of Georgia, Georgia Regents University, \\ Augusta, Georgia, USA \\ pbuckley@gru.edu
}

Relapse in schizophrenia remains a major public health concern. Antipsychotic medications reduce relapse, although the vexing problem of noncompliance greatly influences the illness trajectory. To that end, efforts to detect noncompliance and/or to give medications in a more assured route are central to reducing relapse. It is surprising, then, that recent relapse-prevention studies have not demonstrated any appreciable benefit for long-acting injectable antipsychotics over oral medications. This appears somewhat counterintuitive to clinical experience, wherein longacting injectable antipsychotic medications can stabilise recurrent relapses among patients who are inadequately compliant with medications and have multiple relapses thereupon. This seemingly disparate finding between research relapse prevention trials and clinical practice likely reflects, in part, the requirement that longterm relapse prevention trials must inherently seek sustained commitments from patients. Yet patients who might benefit most from long-acting injectable antipsychotic treatments are less likely to consent to or remain in such trials. Also, the extent of patient engagement and monitoring in relapse prevention trials may enhance compliance, which obfuscates interpretation of such studies. In a 30-month relapse prevention study, we found no overall significant differences in relapse or hospitalisation between the long-acting and oral antipsychotic medication groups. However, there are also studies in first-episode patients that show benefis of medications upon relapse earlier in the illness. This presentation will focus on relapse prevention and cover the available data to date, as well as clinical decision making regarding use of antipsychotic medications to reduce relapse in schizophrenia.

\section{Informed consent in biological treatments: The right to know and the duty to inform I Chetty}

Psychiatrist in Private Practice

indh@hotmail.com

The clinician/patient relationship lies at the heart of the interactions between clinicians, their patients and patient families and caregivers. One of the essentials of a successful relationship between clinician and patient is mutual trust. Inherent in this relationship are principles of law, ethics and public policy. The Constitution of the Republic of South Africa is the supreme law in the country, and all contact has to stand up to constitutional scrutiny. This includes how clinicians conduct themselves in the clinical setting. In compliance with Constitutional principles, the clinical setting can no longer assume a patriarchal interarchical approach with the clinician at the head. Rather, the modern clinical setting is based on a collaborative 
approach reflecting rules of information-sharing and combined decision making. Central to this approach is the concept of informed consent. In addition to the Constitution, other pieces of subordinate legislation and other law and guidelines provide guidance with regard to informed consent. These include the National Health Act, the Mental Health Care Act, the common law and the Health Professionals Council of South Africa Guidelines. It is important to note that ignorance of the law is not a defense for wrongful conduct. It is therefore expected that clinicians themselves follow the relevant law and guidelines in upholding the requirement for informed consent. It is necessary that patients consent prior to the initiation of biological treatments. Attached to the playees in the clinician/patient relationship are certain rights and duties that guide the decision making process leading to informed consent. The patients' rights can be limited in certain circumstances. This is especially relevant to psychiatry in the setting of involuntary care. Informed consent represents a potential minefield, but can be negotiated effectively with careful adherence to the law and good standards of clinical care.

\section{Effectiveness of a long-acting injectable antipsychotic plus an assertive monitoring programme in first-episode schizophrenia}

B Chiliza, ${ }^{1 \star}$ L Asmal, ${ }^{1}$ O Esan, ${ }^{2}$ A Ojagbemi, ${ }^{2}$ O Gureje, ${ }^{2}$ R Emsley ${ }^{1}$

${ }^{1}$ Department of Psychiatry, Stellenbosch University, Cape Town, South Africa

${ }^{2}$ Department of Psychiatry, College of Medicine, University of Ibadan, Ibadan, Nigeria

•bonga@sun.ac.za

Background. Depot antipsychotics were developed in the 1960s to address the problem of adherence in schizophrenia. However, despite their previous widespread use, studies have failed to unequivocally demonstrate their superiority over oral antipsychotics. It can be argued that the greatest benefits of depot antipsychotics would be observed in the earlier phase of illness, in line with the public health principles of early intervention and prevention of accruing morbidity.

Methods. We conducted an open-label trial of a low-dose conventional depot antipsychotic, flupenthixol decanoate, plus an assertive monitoring program in Cape Town, South Africa and Ibadan, Nigeria. We enrolled 207 participants with schizophreniform disorder, schizophrenia and schizoaffective disorder and treated them for 12 months. Clinical variables were measured using the Positive and Negative Syndrome Scale (PANSS), the Clinical Global Impression Severity Scale (CGI-S), the Calgary Depression Scale for Schizophrenia (CDSS) and the Extrapyramidal Symptom Rating Scale (ESRS).

Results. Acceptance of depot treatment plus assertive monitoring programme was good. One hundred and forty-nine (72\%) participants completed the 12 months of treatment. One hundred and four patients achieved full remission at 12 months. This represents $50 \%$ of the entire sample, and $70 \%$ of the patients who remained in treatment throughout. Mean ( \pm standard deviation) maximum increase in ESRS Parkinsonism subscale was 1.5 \pm 1.2 . There were no cases of persistent dyskinesia. There was a substantial increase in body weight in 108 (52\%) patients, with weight gain of $5 \mathrm{~kg}$ or greater.

Conclusion. This study suggests that a low-dose depot antipsychotic plus assertive monitoring is acceptable and feasible in resourceconstrained settings.

\section{Name, shame, fame \\ P Cilliers}

Psychiatrist in Private Practice

practice@plcilliers.co.za

A Medline and Google search for literature addressing bipolar mood disorder (BPD) and creativity is discussed. This topic was precipitated by a paper by J le Blas, 'BPD and Prestige', at the International Review of Bipolar Disorder in Seville, Spain in March 2013. Three historical figures are used as metaphorical examples of creative people with BPD. A large Swedish study linking BPD and creativity, as well as a British Journal of Psychiatry editorial by Kay Jamieson are highlighted. Ethical and practical conclusions are discussed.

\section{Can we manage the increasing incidence of violent raging children? We have to! \\ H Clark}

Child, Adolescent and Family Unit, Chris Hani Baragwanath Hospital, Soweto, Johannesburg, South Africa

hmclark@mweb.co.za

When a violent, uncontained and raging child presents to a paediatric casualty or inpatient setting, it represents an acute child psychiatric emergency. Immediate intervention is required to protect other children, adults, staff and the integrity of the physical space, as well as the safety of a very vulnerable index child. A fairly small proportion of these children fulfill criteria for bipolar rages. Many, particularly in our experience, fall into the category of severe reactive attachment disorder with comorbid conduct disorder. These children often have a background of abuse, trauma and neglect, and are driven by an intense deep seated anger. The practitioner, often in an under-resourced, inadequately staffed facility, has to make an immediate decision on what to do. Chemical restraint may be the only option available, and one has to decide on which drug to use, what dose, as well as the route of administration - all within an acute crisis situation. Delay in taking action and inadequate dosing, necessitating repeated attempts, will lead to an intensification of the agitation, increasing the risk of violence. These are major drugs that have significant potential for side effects, particularly given the required dose and route of administration. This is an area of child psychiatry that can be very intimidating to the practitioner. No two cases are the same. However, these are very distressed children, who deserve the best possible intervention, and one which would befit any other acute paediatric emergency. 
Serotonin, depression and antidepressant action

P Cowen

Department of Psychiatry, University of Oxford, Oxford, UK

phil.cowen@psych.ox.ac.uk

Approximately 50 years ago, the first clinically useful antidepressants were discovered by chance or, more accurately, by brilliant clinical observation. Subsequent laboratory studies revealed that the therapeutic effects of antidepressant agents were probably attributable to potentiation of the monoamine neurotransmitters, serotonin (5-HT) and noradrenaline (NA). This finding has been followed by much human investigation, confirming that patients with depression have abnormalities in monoamine function, particularly in 5-HT mechanisms. Two main questions are raised by these data: (i) Are functional serotonin deficiencies involved in the pathophysiology of depression? (ii) By what mechanisms are the 5-HT potentiating effects of antidepressant drugs translated into clinical therapeutic activity? The role of serotonin in the pathophysiology of depression has been addressed using the technique of tryptophan depletion, which diminishes the availability of the amino acid tryptophan to the brain and lowers brain 5-HT synthesis. In recovered depressed patients tryptophan depletion produces brief symptomatic clinical relapse, together with changes in regional cerebral blood flow in brain areas involved in emotional processing. These findings suggest that in those at risk of depression, diminished 5-HT neurotransmission is sufficient to produce clinical depressive symptomatology. The actions of serotonergic antidepressants have been studied in detail at the molecular level in animal experimental studies, but until recently we have lacked knowledge of the neuropsychological mechanisms that translate enhanced serotonin neurotransmission into clinical antidepressant activity. However, it is now apparent that serotonergic antidepressants produce rapid behavioural and neural changes in the processing of emotional information prior to improvement in subjective mood. For example, the selective serotonin reuptake inhibitor (SSRI) citalopram decreases the processing of negative facial expressions and also attenuates the amygdala response to fear in both healthy volunteers and depressed patients. These findings suggest that the ability of serotonergic antidepressants to help depressive symptoms probably stems from an action on the implicit processing of emotional information rather than a primary effect on mood. This raises the interesting possibility that antidepressants and cognitive behaviour therapy might work in similar ways to relieve depression. Antidepressants could produce positive emotional biases at a 'non-conscious' level in the limbic system, while cognitive behaviour therapy modifies the way that emotional information is explicitly appraised and evaluated.

Prevalence and correlates of comorbid psychiatric illness in patients with heroin use disorder admitted to Stikland Opioid Detoxification Unit

L Dannatt, ${ }^{*}$ KJ Cloete, M Kidd, L Weich

Department of Psychiatry, Faculty of Medicine and Health Sciences,

Stellenbosch University, Cape Town, South Africa

*lisadannatt@yahoo.com
Background. This presentation focuses on the prevalence and correlates of psychiatric comorbidity among patients with heroin dependence admitted to the Opioid Detoxification Unit at Stikland Hospital, Western Cape Province, South Africa.

Method. Participants $(N=141)$ were assessed for psychiatric illness (using the Mini International Neuropsychiatric Interview), comorbid substance use disorders (using the World Health Organization's Alcohol Smoking Substance Involvement Screening Tool), health risk behaviors, health problems, plus personal and social functioning (using the Maudsley Addiction Profile). Data on demographic, personal, past medical, psychiatric, and substance use history; severity of withdrawal symptoms; and mental state examination on admission were collected from the case notes.

Results. Of the 141 patients who took part in this study, the majority were male, single, and with an average level of education of 9 years. Over three-quarters of the participants were unemployed and $14 \%$ of females were pregnant. Most patients were HIV negative based on self-reports. Participants presenting to treatment had on average begun to engage in substance use in their early teens, with cannabis and then methamphetamine as the most commonly used debut drugs. Psychiatric comorbidities were common in this patient sample with regards to both current and previous lifetime prevalence. The most common current comorbidity in this study was major depressive disorder followed by post-traumatic stress disorder (PTSD), social phobia and panic disorder. Previous, lifetime comorbidities included a similar spectrum of major depressive disorder, PTSD and panic disorder. An unexpected finding was that many patients reported previous short-lived psychotic episodes; psychosis is not an effect of heroin use. Most of these patients were able to subsequently link methamphetamine and cannabis use with these episodes. With regards to comorbid substance use, nearly all patients in this study were dependent on nicotine; co-dependency rates for depressants were highest for alcohol, cannabis and methaqualone. The most common stimulant of abuse was methamphetamine. Social functioning was generally poor and drug-related crime was very common, with a majority of participants having been arrested.

Conclusion. Psychiatric comorbidity is prevalent among patients with heroin use disorders in the Western Cape, South Africa. This needs to be considered when planning services for patients with heroin use disorders.

\section{Investigating the association between diabetes mellitus, depression and psychological distress in a cohort of South African teachers}

AK Domingo, ${ }^{*}$ S Seedat, T M Esterhuizen, C Laurence,

J Volmink, L Asmal

Faculty of Medicine and Health Sciences, Stellenbosch University,

Cape Town, South Africa

*abduldomingo@gmail.com

Background. Cardiovascular disease is the second leading cause of death in South Africa, after HIV. Diabetes may increase the risk of depression due to a sense of threat of debilitating complications or due to associated lifestyle changes. Depression may increase the risk of type 2 diabetes due to poor health behaviours. 
Objective. To determine the association between diabetes mellitus, depression and psychological distress in a cohort of South African teachers.

Methods. Teachers from 111 public schools in the Metro South District of the Cape Metropolitan area were invited to participate in this study. Teachers were provided with questionnaires that focused on demographic details and cardiovascular diseases and their risk factors. The Center for Epidemiologic Studies-Depression Scale (CESD) and the Kessler Psychological Distress Scale (K10) were used to assess for depression and psychological distress respectively. A trained nursing sister completed a physical examination. Glucose, cholesterol, and serum creatinine were measured in non-fasting blood samples. Pearson's chi-square tests were used to assess for associations between categorical variables and outcomes, and $t$-tests for continuous variables. Binary logistic regression modeling was carried out to adjust for confounding variables.

Results. Of the 366 teachers that completed the questionnaires, 67.8\% were female and the mean average age \pm standard deviation (SD) was $46.86 \pm 8.62$ years. The mean \pm SD body mass index (BMI) $\left(\mathrm{kg} / \mathrm{m}^{2}\right)$ fell into the 'obese' range (mean $31.23 \pm 7.40$ ), systolic blood pressure mean was $135.01 \pm 19.17 \mathrm{mmHg}$ and diastolic $79.51 \pm 11.28 \mathrm{mmHg}$. Psychological distress was identified in $27.6 \%$ of the cohort and depression in $14.2 \%$. A diagnosis of diabetes mellitus was associated with an increased risk of depression (adjusted odds ratio $(\mathrm{OR})=3.51$, 95\% confidence interval (CI) 1.643 - 7.512) and psychological distress (adjusted OR=2.60, 95\% CI 1.325 - 5.119).

Conclusion. The high prevalence of obesity, diabetes mellitus and high mean systolic blood pressure is concerning in this cohort of South African teachers. These comorbidities often cluster, increasing the risk of metabolic syndrome. A diagnosis of diabetes mellitus was strongly associated with an increased risk of depression and psychological distress. The interrelationship between anthropometric, psychological, lifestyle and metabolic factors needs further investigation.

\section{Neuropeptide $S$ - emerging evidence for a role in anxiety K Domschke \\ Department of Psychiatry, University of Wuerzburg, Germany domschke_k@klinik.uni-wuerzburg.de}

Animal models, as well as recent molecular genetic, imaging genetic, gene-environment interaction and psychophysiological studies, implicate the neuropeptide S (NPS) system as a novel pathomechanism in the aetiology of anxiety and panic disorder in particular. In several independent studies, a functional $\mathrm{A} / \mathrm{T}$ single nucleotide polymorphism (rs324981) causing an amino acid exchange from Asn to Ile (N107I) in the neuropeptide S receptor gene (NPSR) has been reported to be associated with panic disorder, particularly in female patients. In a gene-environment (GxE) interaction study, a significant interactive GxE effect of the NPSR A/T polymorphism and childhood maltreatment (CTQ) on anxiety sensitivity (AS) was identified: carriers of the more active NPSR T/T genotype who had had a high number of maltreatment experiences in childhood reported increased anxiety sensitivity. On a neural network level, an imaging genetic study yielded evidence for the more active NPSR $\mathrm{T}$ allele to be associated with decreased activity in the dorsolateral prefrontal, lateral orbitofrontal and anterior cingulate cortex during processing of anxiety-relevant emotional stimuli in patients with panic disorder. The NPSR T risk allele was furthermore found to be related to increased heart rate as well as higher symptom reports and elevated anxiety sensitivity during a behavioral avoidance test, as peripheral neurophysiological and dimensional intermediate phenotypes of anxiety, respectively. In extension of previous evidence from rodent studies, a multilevel human study provided support for a complex, non-linear interaction of the neuropeptide S (NPSR T/T genotype) and adenosinergic (caffeine challenge paradigm) systems affecting the affect-modulated startle response as an intermediate phenotype of anxiety in humans. Novel findings suggest an influence of NPSR A/T on cognitive response control processes such as error monitoring and response inhibition as measured by electroencephalography. In summary, the present body of research provides converging lines of evidence for a role of NPSR gene variation in the pathogenesis of anxiety. The more active NPSR rs324981 T allele (Ile107) has been found to be associated with the categorical phenotype of panic disorder in a female-dominant manner, possibly intertwined with alterations in the adenosinergic system, in interaction with early trauma and mediated by elevated anxiety sensitivity, increased autonomic arousal as well as distorted processing of fear-related emotional stimuli. In synopsis with previous evidence from animal models, these findings might nourish future studies exploring a potentially beneficial use of therapeutic agents targeting the NPS system in anxiety disorders.

\section{Pathogenetics of anxiety \\ K Domschke \\ Department of Psychiatry, University of Wuerzburg, Germany \\ domschke_k@klinik.uni-wuerzburg.de}

Anxiety disorders constitute the most frequent mental disorders worldwide, with a lifetime prevalence of about $15 \%$, and confer a high socioeconomic burden. The pathogenesis of anxiety disorders is multifactorial, with an interaction between biological and environmental factors. Among biological factors, genetic mechanisms play a particularly important role in the pathogenesis of anxiety disorders, with clinical genetic studies suggesting a heritability of about $30-67 \%$. The present talk will give an overview of linkage studies, association studies and genome-wide association studies (GWAS) yielding support for candidate loci and (partly femalespecific) risk genes of anxiety. Additionally, first evidence for gene-environment interactions between candidate genes of anxiety disorders and stressful life events will be reported. On a systems level, neural activation correlates of anxiety-relevant emotional processing and neurophysiological measures such as peripheral sympathetic activity or the startle reflex have been shown to be potentially driven by vulnerability genes of anxiety disorders. Pharmaco- and psychotherapy-genetic studies provide evidence for certain risk genes to confer interindividual variability in response to therapeutic interventions in anxiety disorders. Promising approaches to further dissect the genetic underpinnings of anxiety disorders such as epigenetic analyses will be presented. Genetic research in anxiety disorders will be discussed with respect to its potential benefit 
for future efforts to develop innovative and individually tailored therapeutic approaches for patients with anxiety disorders.

\author{
The effects of HIV on the fronto-striatal system \\ $\underline{\text { S du Plessis }},{ }^{1 \star}$ M Vink, ${ }^{2}$ J Joska, ${ }^{5}$ E Koutsilieri, ${ }^{3}$ C Scheller, ${ }^{3}$ \\ B Spottiswoode, ${ }^{6}$ D Stein, ${ }^{4,5}$ R Emsley ${ }^{1}$ \\ ${ }^{1}$ Department of Psychiatry, Stellenbosch University, Cape Town, \\ South Africa \\ ${ }^{2}$ Rudolf Magnus Institute of Neuroscience, University of Utrecht, \\ The Netherlands \\ ${ }^{3}$ Institute for Virology and Immunobiology, University of Würzburg, \\ Würzburg, Germany \\ ${ }^{4}$ Medical Research Council Unit on Anxiety and Stress Disorders, \\ Cape Town, South Africa \\ ${ }^{5}$ Department of Psychiatry, University of Cape Town, South Africa \\ ${ }^{6}$ Medical Research Council/University of Cape Town Medical \\ Imaging Research Unit, Department of Human Biology, University of \\ Cape Town, South Africa \\ *stefandup@sun.ac.za
}

Background. The milder forms of HIV-associated neurocognitive disorders (HANDs) are potentially reversible with early treatment. Yet HAND remains difficult to diagnose, as our understanding of this subcortical dementia remains limited.

Objective. To investigate the effects of HIV on cortical and subcortical components of the fronto-striatal system during inhibitory control.

Method. 18 HIV-positive participants (mean age 30 years; mean $\mathrm{CD}^{+} 376$ cells/ $\mu$; with cognition unimpaired to moderately impaired; on highly active antiretroviral therapy $n=2$ ) and 17 gender, age, education and ethnically matched controls underwent functional magnetic resonance imaging (fMRI). Participants were required to press a button on go-signals (go response) and refrain from pressing it on intermittent stop-signals (inhibition). These go responses, in the absence of stop-signals were used to measure basic motor function. 'Reactive inhibition', a sub-cortically driven process, is represented by the correct inhibition of a go response in reaction to a stop-signal. This was assessed by calculating participant speed of inhibition (SSRT). On fMRI this was investigated by comparing successful stop-signal trials with incorrect stop-signal trials. 'Proactive inhibition' was also assessed, as stop-signal probability was explicitly stated, allowing subjects to anticipate stop-signals and slow down their response times proactively. This form of cortical inhibition was measured by correlating proactive response time slowing with increases in stopsignal probability. On fMRI this was measured by correlating increases in stop-signal probability with go-signal activation.

Results. HIV-positive participants were significantly slower on 'go' baseline trials than controls $(p=0.003)$. Reactive inhibition: SSRT did not differ between the groups $(p=0.835)$. fMRI revealed bilateral putamen hypoactivation in the HIV-positive group. Proactive inhibition: proactive response slowing did not differ between the groups ( $p=0.073$ ). The HIV-positive group showed hyperactivation in the anterior cingulate cortex.

Conclusion. Subjects showed baseline go response slowing, in keeping with subcortical pathology. Despite similar behavioural performance on measures of inhibition, HIV-positive participants showed subcortical hypoactivation and cortical hyperactivation. A potential explanation for this is that HIV primarily impacts subcortical areas and the cortex is able to compensate for this in the milder forms of HAND, given the similar performance on our cortical measures. However, larger prospective clinical fMRI studies are needed to confirm these findings.

\section{Effects of acute antipsychotic treatment on brain morphology in schizophrenia \\ R Emsley, ${ }^{\star}$ L Asmal, B Chiliza, S du Plessis, J Carr, A Goosen, M Kidd, M Vink, R Kahn \\ Department of Psychiatry, Faculty of Medicine and Health Sciences, Stellenbosch University, Cape Town, South Africa \\ *rae@sun.ac.za}

Background. Progressive global and regional structural brain changes have been reported in schizophrenia, even in the course of acute treatment. It is not clear whether they reflect illness progression, direct effects of antipsychotic medication, or secondary factors such as substance abuse, poor adherence to treatment or comorbid conditions. Methods. Twenty-two antipsychotic-naive patients with a firstepisode of schizophrenia, schizophreniform or schizoaffective disorder received treatment over 13 weeks with either risperidone long-acting injection or flupenthixol decanoate in a randomised, double-blinded design, and were compared with 23 matched healthy volunteers. T1-weighted data on a $3 \mathrm{~T}$ magnetic resonance imaging scanner were obtained at weeks 0,4 and 13. Images were reconstructed using a validated whole-brain segmentation technique which included a longitudinal processing stream. Treatment response was assessed by changes in psychopathology, functionality, quality of life and cognitive performance. Tolerability was assessed by motor disorders, serum prolactin, weight, and fasting glucose and lipid profiles.

Results. At baseline, patients demonstrated reduced cortical thickness in several regions bilaterally, larger left inferior lateral ventricular volume, and larger bilateral caudate, left thalamus and posterior corpus callosum volumes compared with controls. Compared with healthy controls, the patients exhibited significant longitudinal reductions in 2 measures of temporal cortical thickness and the ventral diencephalon bilaterally, and increased fourth ventricular volume in the primary analysis. Post hoc correlational analyses suggest that some brain changes are associated with improvements in psychopathology and quality of life, and others with emergent motor disorders, weight gain and changes in lipid profile.

Conclusion. Our data provide further evidence that changes occur in multiple brain regions with acute antipsychotic treatment, and suggest that some of these changes are related to treatment response and others to emergent treatment side-effects.

\section{Development of a genetic database resource for monitoring of breast cancer patients at risk of physical and psychological complications}

K Grant,${ }^{1,5 \star}$ F J Cronje, ${ }^{2}$ K Botha, ${ }^{3}$ J P Apffelstaedt, ${ }^{4}$ M J Kotze ${ }^{5}$ 
${ }^{1}$ Department of Biomedical Sciences, Faculty of Health and Wellness, Cape Peninsula University of Technology, Bellville, South Africa ${ }^{2}$ Department of Interdisciplinary Health Science, Faculty of Health and Medicine, University of Stellenbosch, Tygerberg

${ }^{3}$ Paarl Medical Centre, Paarl, South Africa.

${ }^{4}$ Department of Surgery, Faculty of Health and Medicine, University of Stellenbosch, Cape Town, South Africa

${ }^{5}$ Department of Pathology, Faculty of Health and Medicine, University of Stellenbosch, Cape Town, South Africa

*grantk@cput.ac.za

Background. Although recent advances in treatment for breast cancer have improved survival rates, psychological morbidity remains a significant concern. Depression and anxiety are common complications and are exacerbated by the prospect of chemotherapy and the potential for tumour recurrence. A newly developed MammaPrint Prescreen Algorithm (MPA) to identify patients eligible for the 70-gene profile has allowed $\sim 60 \%$ of early-stage breast cancer patients to forego chemotherapy due to greater confidence in long-term survival. The need to monitor health outcomes in breast cancer patients and obtain prospective data has been identified as an important priority.

Methods. In response to a needs assessment survey amongst healthcare professionals in South Africa, a pathology supported genetic testing (PSGT) service was established for breast cancer patients and linked to a health outcomes research project. The family history, clinical information, genetic and pathology test results of 150 breast cancer patients were captured using a secure online database. The genetic component included results from (i) the 70-gene MPA profile, (ii) BRCA1/2 mutation detection and (iii) a comprehensive wellness screen including CYP2D6, COMT and MTHFR genotyping. Results. Analysis of patient data permitted comprehensive risk profiling for targeted health monitoring over time. A representative case, who underwent all 3 types of genetic tests due to a strong family history and bilateral tumours, was designated low-risk MPA, BRCA negative, with a functional CYP2D6 gene supporting the use of standard tamoxifen doses. However, genetic risk factors associated with a dysfunction of the methylation pathway and oestrogen metabolism were identified in both the index case and her daughter with breast cancer.

Conclusion. MPA may allow up to $60 \%$ of patients to forego chemotherapy, but consideration of additional information as part of a wellness screen may facilitate early identification of patients at increased risk of physical and psychological complications. PSGT using an integrated database for collating clinical information relevant to the laboratory tests performed ( $i$ ) adds confidence in conservative treatment decisions, (ii) combines these results with genetic data for more effective health monitoring, and (iii) offers an accelerated translational research opportunity that may benefit breast cancer patients and their family members.

\author{
Unipolar mania reconsidered: Evidence from a \\ South African study \\ C Grobler \\ Department of Psychiatry, Walter Sisulu University, Mthatha, \\ South Africa \\ dr.stof@mweb.co.za
}

Background. There is a lack of studies that examine the prevalence and phenomenology of bipolar disorder in Africa. In literature, a unipolar manic course of illness in particular is reported to be rare. Objectives. To investigate and describe the course of illness and clinical features for a cross-section of patients diagnosed with bipolar disorder attending public hospitals in Limpopo Province, South Africa, and to determine the rate of a unipolar manic course in this sample of patients. Method. This was a descriptive, cross-sectional study of patients presenting with a history of mania between October 2009 and April 2010, to 3 hospitals in Limpopo Province. A purposeful sample of 103 patients was recruited and interviewed using the Affective Disorders Evaluation.

Results. This study confirms that a unipolar manic course is indeed much more common than occurrences suggested in present day literature, with $57 \%$ of the study sample ever experiencing manic episodes. Patients presenting with a unipolar manic course of illness, as described in this study, may contribute to the search for an aetiologically homogeneous subgroup which presents a unique phenotype for genetic research and the search for genetic markers in mental illness. With a view to future research, a unipolar manic course therefore needs to be considered as a specifier in diagnostic systems in order to increase the awareness of such a course of illness in bipolar disorder.

Conclusion. Fifty-seven percent of study subjects had only ever experienced manic episodes which is in keeping with findings from Africa and other non-Western countries. Identifying aetiologically homogenous subgroups in psychiatry can also aid the profession in developing a reliable and valid nosology for psychiatric disorders. We need to consider a unipolar manic course at least a specifier in the Diagnostic and Statistical Manual of Mental Disorders and the International Classification of Diseases.

\section{Antipsychotic-induced movement disorders: Occurrence and management \\ $\underline{\text { P Haddad }}^{1,2}$}

${ }^{1}$ Consultant Psychiatrist, Greater Manchester West Mental Health NHS Foundation Trust, UK

${ }^{2}$ Honorary Reader, Neuroscience and Psychiatry Unit, University of Manchester, UK

peterhaddad@doctors.org.uk

This presentation will review the main extrapyramidal syndromes (EPS) seen with antipsychotic drugs, explore the issue of differential risk with specific drugs and conclude by considering management. The main EPS are parkinsonism, acute dystonia, akathisia and tardive dyskinesia (TD) and their prevalence and impact is often underestimated. The risk of EPS varies by individual drug and not by 'class'. A spurious dichotomy of antipsychotic drugs into first- and second-generation agents masks the differential risk of EPS and other side-effects. Various mechanisms can contribute to low EPS risk, including low affinity and fast dissociation from D2 receptors, M1 blockade and partial agonism at D2 receptors. Management centres on reducing the risk of occurrence, via prescribing choices, early detection of EPS by screening and offering appropriate intervention. Interventions for EPS, other than TD, include decreasing the antipsychotic dose, offering 
specific treatments (e.g. an anticholinergic agent for parkinsonism) and switching to an antipsychotic with lower EPS-risk. It is not possible to give an evidence-based treatment algorithm for TD. The conventional view is to stop antipsychotic medication if possible, or to reduce the dose or switch to an antipsychotic with low EPS-risk, but these approaches are not supported by randomised controlled trials. A wide range of anti-dyskinetic medications have been suggested, but there is no robust evidence base for any of them. The most common outcome for TD, irrespective of treatment, is persistence with modest fluctuation in severity, and among other patients there is an equal chance for improvement or worsening.

\section{The place of observational studies in assessing the effectiveness of long-acting injectable antipsychotics P Haddad, ${ }^{1,2}$ \\ ${ }^{1}$ Consultant Psychiatrist, Greater Manchester West Mental Health NHS Foundation Trust, UK \\ ${ }^{2}$ Honorary Reader, Neuroscience and Psychiatry Unit, University of Manchester, UK \\ peterhaddad@doctors.org.uk}

Most well-conducted observational studies report better clinical outcomes (longer continuation rates and/or reduced hospitalisation rates) for antipsychotic long-acting injections (LAI) compared with oral antipsychotics. Of particular note are studies comparing the same antipsychotics in oral and LAI form, which overcome efficacy differences between antipsychotics influencing outcome. In a nationwide observational study from Finland, the risk of rehospitalisation after an initial hospitalisation for schizophrenia patients receiving LAI was about one-third of that for patients receiving equivalent oral agents. This is despite prescribing bias tending to favour using LAIs in more relapse-prone patients. In contrast, randomised controlled clinical trials (RCTs) tend to show equivalent relapse rates between oral antipsychotics and LAIs, though some studies have shown some relative benefit for LAIs in secondary outcomes. No RCT has shown a worse outcome with long-acting medication. In this presentation the observational and RCT data will be reviewed and possible explanations for the differences explored. It will be argued that methodological problems with current RCTs, relating to cohort bias and the validity of treatment delivery, mean that they may not be the 'gold standard' in this area. Observational studies have their own methodological weaknesses but at the very least a full assessment of the evidence base for long-acting antipsychotic formulations should consider observational data as well as RCTs.

\footnotetext{
Molecular mechanisms of d-cycloserine in fear extinction:

Insights from RNA sequencing

$\underline{\text { S Hemmings, }}{ }^{1 *}$ S Malan-Müller, ${ }^{1}$ L Fairbairn, ${ }^{1}$ M Jalali, ${ }^{2}$

E J Oakeley, ${ }^{4}$ J Gamieldien, ${ }^{2}$ M Kidd, ${ }^{3}$ S Seedat ${ }^{1}$

${ }^{1}$ Department of Psychiatry, Stellenbosch University, Cape Town, South Africa

${ }^{2}$ South African National Bioinformatics Institute, University of the Western Cape, Cape Town
}

\author{
${ }^{3}$ Centre for Statistical Consultation, Stellenbosch University, \\ Cape Town, South Africa \\ ${ }^{4}$ Novartis Institutes for BioMedical Research, Biomarker Development, \\ Human Genetics and Genomics, Genome Technologies, Basel, \\ Switzerland \\ *smjh@sun.ac.za
}

Background. Post-traumatic stress disorder (PTSD) is a severe, chronic and debilitating psychiatric disorder that can occur after a traumatic event. D-cycloserine (DCS), a partial N-methyl-D-aspartate (NMDA) receptor agonist, was shown to be effective in facilitating fear extinction in animal and human studies of anxiety. The precise mechanism whereby DCS facilitates fear extinction is unknown.

Objective. To elucidate the molecular mechanism of action of DCS in facilitating fear extinction in a rat model of PTSD.

Methods. Adult Sprague-Dawley rats were grouped into 4 experimental groups (30 rats/group), with saline or DCS infused intrahippocampally and one half receiving an electric foot shock. Animals were subjected to behavioural tests to determine which displayed anxiety-like behaviour. RNA was extracted from the left dorsal hippocampi (LDH) and was used for RNA sequence analysis. Differentially expressed genes between different treatment groups were identified using bioinformatics analyses. Suitability of quantitaive polymeric chain reaction ( $\mathrm{qPCR}$ ) as verification analysis of sequencing data was also investigated.

Results. The focus experimental groups were fear + saline maladapted $($ FSM ) v. fear + DCS well-adapted (FDW) in order to identify DCSinduced fear extinction gene expression changes. One hundred and eight genes were significantly down-regulated in the FDW group compared with the FSM group, and 39 genes were predicted to be biologically significant based on their function. Subsets of differentially expressed genes were common between memory disorders, nervous system diseases, metabolic disorders and substance- and alcohol-related disorders; these included Trh, Mmp9, Mt2a, Clec7a, Il1rn, Npy, Spp1 and Cybb. Relative expression analyses with qPCR detected 3 differentially expressed genes, Cybb, Mt2a and Illrn, (from a panel of 8 selected genes) in the brain cDNA and 1 gene, matrix metallopeptidase 9 (Mmp9), in the blood cDNA.

Conclusions. Differential gene expression analyses in this PTSD animal model enabled us to identify genes, networks and pathways that might explain how DCS facilitates fear extinction. Furthermore, differentially expressed genes that were common in different diseases might help to explain the comorbidity described for these diseases. Identifying the molecular underpinnings of fear extinction might bring us closer to understanding and effectively treating PTSD.

\section{Schizophrenia: The role of inflammation DC Henderson \\ Department of Psychiatry, University of Montreal, Quebec, Canada dchenderson@partners.org}

The activation of inflammatory pathways in the central nervous system (CNS) has been proposed as one of the potential mechanisms contributing to the aetiology of schizophrenia. Previous research has 
demonstrated that prenatal exposure to infections, especially in the third trimester of pregnancy, is associated with an increased risk of schizophrenia in the offspring. Furthermore, there has been evidence linking obstetric complications to schizophrenia. A five-fold increased risk for developing psychoses later on was detected after infection of the central nervous system in early childhood. The levels of several proinflammatory factors and cytokines have all been shown to be increased in schizophrenia patients. Studies of antipsychotic-naïve patients with first-episode psychosis have found that inflammation is present already at this stage. Some of these abnormalities resolve after the initiation of treatment, suggesting that they may be state markers of acute psychosis, but other abnormalities persist. Furthermore, post mortem immunohistochemical investigations showed enhanced microglia densities in the brains of schizophrenia patients, and other studies using positron emission tomography (PET) confirmed microglia activation. In parallel, there is a separate body of evidence relating subclinical chronic inflammation and schizophrenia in individuals, usually in their adulthood, who have already developed schizophrenia. On the other hand, unequivocal experimental, epidemiological and clinical evidence has emerged during the past decade linking inflammation to the development of insulin resistance and metabolic disturbances, which are common in the schizophrenia population. Inflammation might be an important common pathophysiological process related to both schizophrenia psychopathology and metabolic disturbances seen in patients with schizophrenia. Future studies targeting pro-inflammatory cytokines and their molecular signaling pathways may lead to novel pharmacological intervention strategies treating both psychopathology and medical comorbidity in patients with this devastating mental illness.

\section{Addictions: Emergent trends and innovations \\ V Hitzeroth}

Psychiatrist in Private Practice

lkramer@eccsystems.co.za

The neuroscience of addictions is a rapidly growing and vastly expanding scientific field. Much has been studied and discovered in the last decade. Unfortunately, no unifying model of addiction has yet been proposed. Rather, numerous different hypotheses and theories are vigorously researched and subsequently compete with each other. This presentation will review the current state of addiction neuroscience, and summarise current emergent trends and innovations within the neurobiology of addictions. Several research hypotheses, with their respective models will be presented, informing the listener of the latest neuroscientific advances in the field. The presentation will also weave new treatment opportunities and developments into the neurobiological findings. This presentation will provide an overview of recent neurobiological advances in the addictions field and an evidence-based and rational approach to the future treatments of addictions.

\section{The socio-cultural-religious context of biological psychiatric practice B Janse van Rensburg}

Department of Psychiatry, University of the Witwatersrand, Johannesburg, South Africa

bernardj@gpg.gov.za

Background. Biological psychiatry as a clinical specialty involves the medical study, diagnosis, treatment and prevention of mental illness. Psychiatric disorders are currently classified to include clinical syndromes such as cognitive disorders, substance-related disorders, the neuropsychiatric aspects of a range of medical disorders, schizophrenia, mood and anxiety disorders and others. The study of mental illness includes neuro-anatomy and physiology, psychopharmacology, genetics, neuro-endocrinology, neuro-imaging, etc. Contributions regarding underlying explanatory models have been considered from the basic, medical, psychological and the socio-cultural perspectives. However, practitioners and teachers of biological psychiatry contend daily with the extent to which religion and spirituality in particular play a role in the lives and experiences of the people with whom they deal as patients or as family members.

Methods. A literature review was undertaken of the psycho-socialreligious ('naturalistic') context in which biological psychiatry is practised on a daily basis, to identify the cultural-religious and spiritual issues impacting on clinical psychiatric assessment, diagnosis, management and outcome.

Results. Psychiatry is currently taught and practised in terms of a biopsycho-social model and operates in the context of a multidisciplinary team (including nursing, social work, psychology and occupational therapy) in specialty- and subspecialty-level facilities and services in public and private sectors. Quantitative and qualitative experimental and research methods, including epidemiology, are being employed to investigate the incidence and prevalence of symptoms and conditions, as well as to assess the response to treatment and to develop new medications and interventions. Themes from the literature include the role of culture and religion in adherence to medication and the impact on the pathways to and outcome of psychiatric care and treatment.

Conclusion. Psychiatry seems to be uniquely positioned in a 'boundary' or 'gate-keeping' role, being an inherent part of the scientific evidencebased fraternity of clinical medicine, while at the same time, in every day practice, also having to consider, interpret, translate and manage scientific knowledge and interventions in a real-life world with multilayered social, cultural/political and religious/spiritual domains.

\section{Biochemical markers for identifying risk factors for disability progression in multiple sclerosis}

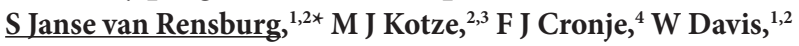

K Moremi, ${ }^{1,2}$ M Jalali Sefid Dashti, ${ }^{5}$ J Gamieldien, ${ }^{5}$ D Geiger, ${ }^{2,3}$

M Rensburg, ${ }^{1,2} \mathrm{R}$ van Toorn, ${ }^{6} \mathrm{M}$ J de Klerk, ${ }^{1,2} \mathrm{G}$ M Hon, ${ }^{7}$ T Matsha, ${ }^{7}$

S Hassan, ${ }^{7}$ R T Erasmus ${ }^{1,2}$

${ }^{1}$ Department of Chemical Pathology, Stellenbosch University,

Cape Town, South Africa

${ }^{2}$ National Health Laboratory Service, Cape Town, South Africa

${ }^{3}$ Department of Anatomical Pathology, Stellenbosch University,

Cape Town, South Africa

${ }^{4}$ Interdisciplinary Medicine, Stellenbosch University, Cape Town, South Africa 
${ }^{5}$ Knowledge Integration \& Biomarker Discovery, South African National Bioinformatics Institute, University of the Western Cape, Cape Town, South Africa

${ }^{6}$ Paediatric Medicine and Child Health, Stellenbosch University, Cape Town, South Africa

${ }^{7}$ Department of Bio-Medical Sciences, Faculty of Health and Wellness Science, Cape Peninsula University of Technology, Cape Town, South Africa

*sjvr@sun.ac.za

Background. Current therapeutic strategies for multiple sclerosis (MS), based on immune modulation, have modest efficacy in the prevention of disability.

Objective. Pathology supported genetic testing (PSGT) was used to identify risk factors for disability progression and to guide personalised intervention to improve disease outcome in MS.

Methods. Patients with MS $(N=130)$ were assessed using lifestyle and diet questionnaires, as well as biochemical tests to identify markers for disability progression. Disability status was assessed using the Expanded Disability Status Scale (EDSS), ranging from 0 (no disability) to 10 (death due to MS). After following a personalised intervention programme that addressed the identified risk factors, patients were reassessed after 6 months, and again after 7 years.

Results. At baseline, $30 \%$ of patients had non-anaemic iron deficiency, $65 \%$ had raised cholesterol values $(>5.0 \mathrm{mmol} / \mathrm{l})$, and $67 \%$ had high homocysteine levels. Vitamin D deficiency $(<30 \mathrm{ng} / \mathrm{ml})$ was found in $67 \%$ and vitamin $\mathrm{D}$ insufficiency $(<50 \mathrm{ng} / \mathrm{ml})$ in $81 \%$ of patients. In a pilot study with personalised intervention over 6 months, 12 compliant patients showed a significant improvement of $29.9 \%$ (the mean EDSS decreased from 3.50 to 2.45). After 7 years 12 compliant patients had a mean \pm standard deviation (SD) EDSS of 1.4 \pm 0.9 . All of these patients scored $\leq 2.5$, which is regarded as benign MS. Clinical improvement correlated with normalisation of blood parameters. Control subjects $(n=12)$ who were not compliant, had a mean \pm SD EDSS of $8.4 \pm 1.5$ after 7 years (difference significant, $p<0.0001$ ).

Conclusion. Identifying risk factors for disability progression in MS using a PSGT personalised intervention programme is an effective strategy for sustained clinical improvement, and an affordable health option.

\author{
Alcohol-induced psychotic disorder: Brain perfusion and \\ psychopathology - before and after antipsychotic treatment \\ G Jordaan, ${ }^{1 *}$ J M Warwick, ${ }^{2}$ D G Nel, ${ }^{3}$ R Hewlett, ${ }^{4}$ R Emsley ${ }^{1}$ \\ ${ }^{1}$ Department of Psychiatry, Faculty of Health Sciences, Stellenbosch \\ University, Cape Town, South Africa \\ ${ }^{2}$ Department of Nuclear Medicine, Faculty of Health Sciences, \\ Stellenbosch University, Cape Town, South Africa \\ ${ }^{3}$ Centre for Statistical Consultation, Faculty of Health Sciences, \\ Stellenbosch University, Cape Town, South Africa \\ ${ }^{4}$ Department of Anatomical Pathology, Faculty of Health Sciences, \\ Stellenbosch University, Cape Town, South Africa \\ *gpj2@sun.ac.za
}

Background. Alcohol-induced psychotic disorder (AIPD) also known as alcohol hallucinosis, is a rare complication of alcohol abuse. AIPD's pathogenesis and treatment are still unclear. Few prospective treatment studies are available, but case reports suggest that antipsychotic treatment is effective. Brain imaging changes in relation to treatment response also have not been studied except for case reports.

Objective. To investigate the effect of antipsychotic treatment on psychopathology and regional cerebral blood flow ( $\mathrm{rCBF}$ ) in patients with AIPD before and after 6 weeks of treatment.

Methods. Nineteen patients with AIPD were assessed by the Positive and Negative Syndrome Scale (PANSS) and single photon emission computed tomography (SPECT) before and after 6 weeks of openlabel treatment with a fixed dose of haloperidol ( $5 \mathrm{mg} /$ day).

Results. Highly significant improvements were noted on the positive, general and total scores of the PANSS. Post-treatment increased rCBF to the left caudate and left frontal lobe was noted. Changes in frontal, temporal, parietal, occipital, thalamic and cerebellar rCBF showed significant negative correlations with the degree of symptom improvement, suggesting dysfunction of these areas in AIPD.

Conclusion. Psychopathological and $\mathrm{rCBF}$ findings suggest reversible generalised cerebral dysfunction in AIPD.

\section{'Pump and dump': Harm reduction strategies for breastfeeding while using substances \\ L Kramer \\ Private Practice \\ lkramer@eccsystems.co.za}

Within the addictions field there is a sparse research base available to provide guidance for breastfeeding and the drug-dependent woman. This topic is little spoken-about, as women are often simply advised to abstain or do not report their drug use for fear of adverse consequences or stigmatisation. However, there is a subgroup of women who may not be willing or able to abstain and who would need to be provided with harm-reduction strategies while they continue using substances after the birth of their child. This presentation will briefly discuss the physiology of breast milk production. Thereafter it will specifically focus on the clinical implications for breastfeeding while using various substances, including harm-reduction strategies. An approach to the evaluation of the mother-infant dyad and how to offer guidance prior to the institution of breastfeeding will be explored. This presentation will address the approach to the management of the drug-dependent woman who is unwilling or unable to abstain from drug use. (Please note this presentation does not intend to address advising women on the use of prescription medications during breastfeeding: rather, it focuses on illicit drugs and other substances such as alcohol and nicotine.)

\section{Adolescent neuropsychiatry - an emerging field in South African adolescent psychiatric services \\ A Lachman}

Tygerberg Hospital, Department of Psychiatry, University of

Stellenbosh, Cape Town, South Africa

anusha@sun.ac.za

Neuropsychiatry is a rapidly expanding field, linking the traditional areas of neurology and psychiatry, which has benefited greatly from 
recognition of the role of genetic and environmental determinants of psychiatric disorders, particularly in childhood and adolescence (C\&A). Children with neuropsychiatric problems often exhibit complex combinations of emotional, behavioural, and cognitive difficulties that defy simple diagnostic or therapeutic treatment. Early diagnosis and treatment can improve outcomes and prevent unnecessary treatment trials. In C\&A literature, 'neuropsychiatric disorders' refer to the diagnostic clusters of autism spectrum disorders, tic disorders, attention deficit and hyperactivity disorder and anxiety disorders (obsessive compulsive disorders) with known neurobiological substrates and which present with psychiatric complaints. In recent times the availability of neuroimaging and genetic investigations at tertiary psychiatric facilities have allowed for more comprehensive and clinically sound diagnoses in children and adolescents presenting with diagnostically difficult symptoms. A case series of neuropsychiatric conditions, diagnosed at a state adolescent psychiatric inpatient facility, will be discussed, emphasising the diagnostic mimicry that may be present and highlighting the use of laboratory testing (biochemical and genetic) and neuro-imaging techniques to assist diagnosis. The cases include juvenile Huntington's disease, idiopathic hypoparathyroidism, viral encephalitis, HIV dencephalopathy and a rare case of KleineLevin syndrome. All presented with acute psychiatric symptoms warranting emergency admissions. Imaging and genetic findings are explained and clinical lessons are shared. Suggestions on screening and symptom clusters in adolescents are discussed with a view to improving awareness and encouraging investigation of possible neuropsychiatric disorders presenting in the clinical C\&A psychiatric setting.

\section{Recovery versus remission, or what it means to be healthy for a psychiatric patient? \\ $\underline{\text { B Latecki }}^{1,2}$ \\ ${ }^{1}$ Philosophy Department, University of Zululand, Empangeni, South Africa \\ ${ }^{2}$ Empangeni Garden Clinic, Empangeni, South Africa latecki@iafrica.com}

Let us acknowledge that a patient's request, 'I just want to be happy', is one of the most challenging, if not horrifying tasks the psychiatrist may be charged with. While pointing out that lack of symptoms does not constitute health, the author examines the outcome of treatment in psychiatry as it is perceived by the patients and the doctors, the concepts of recovery and remission, and the state of wellbeing as opposed to happiness. Clinical, statistical and philosophical concepts are considered. It is also debated as to what extent the available means of treatment can be of use in achieving 'health' in a patient.

\author{
Holistic methods utilised to normalise behaviours in youth \\ diagnosed with neuro-biochemical disorders \\ $\underline{\text { P Macqueen }}^{1,2}$ \\ ${ }^{1}$ Viva Youth College, Kyalami, South Africa \\ ${ }^{2}$ Viva Youth Centre SDN Bhd for outpatients, Malaysia \\ paola@vivacollege.co.za
}

Objective. To normalise youth diagnosed with neuro-biochemical disorders and re-integrate them into society with a completed education and/or life skill qualification. To stabilise/normalise and reintegrate these students so as to allow them to cope with stresses of society; to build on their strengths toward a life skills interest and offer them the opportunity to become contributing members of society and no longer be a burden to families nor the state.

Results. The age range of participants was 13 - 25 years. Neurobiochemical disorders/disabilities included: schizophrenia; bipolar; attention deficit disorder/hyperactivity disorder; Asperger's syndrome; reactive attachment disorder; personality disorder; oppositional defiance disorder; eating disorders; anxiety and depressive disorders; obsessive compulsive disorder; substance abuse and behavioural disorders in general.

Method. Behavioural holistic methods were utilised to normalise youth. The subjects were young people who exhibited inappropriate behaviours due to their chemical imbalances and environmental influences from birth. The Viva methodology followed a secondary family systems approach which included: behaviour modification techniques; individual, group and family therapeutic interventions; task-related programmes with set goals; dynamic educative and life skills approach; structured plan and long-term maintenance; sports programmes and pet therapy.

Conclusion. With the co-operation of the student and their families, relevant structures and routines in place and medication compliance (where required), without substance use and abuse being present, there is a $70 \%$ possibility of success for these students to lead very normal and regular lives, excelling at what they do best.

\section{Candidate genes and novel polymorphisms for anxiety disorder in a South African cohort \\ N McGregor,${ }^{1 \star}$ J Dimatelis, ${ }^{2}$ S M J Hemmings, ${ }^{1}$ C J Kinnear, ${ }^{3}$ D Stein, ${ }^{4}$ V Russel, ${ }^{2}$ C Lochner ${ }^{1}$ \\ ${ }^{1}$ Department of Psychiatry, Stellenbosch University, Cape Town, South Africa \\ ${ }^{2}$ Department of Physiology University of Cape Town, South Africa \\ ${ }^{3}$ Department of Molecular Biology and Human Genetics, \\ Stellenbosch University, Cape Town, South Africa \\ ${ }^{4}$ Medical Research Council Unit on Anxiety and Stress Disorders, Department of Psychiatry and Mental Health, University of Cape \\ Town, South Africa \\ *nwm@sun.ac.za}

Background. Anxiety aetiology remains poorly understood. However, clear evidence for a genetic component exists. Increasing focus on neurobiological and environmental interaction mediating disorder pathogenesis has been highlighted, specifically the role of trauma in disorder susceptibility. A number of genetic studies have been conducted; however, as yet, no genetic variants have been explicitly identified as being involved in disorder pathogenicity. Neural circuitry involving the striatum has been implicated in numerous anxiety disorders. Identifying mechanisms and genes responsible for changes at neuronal synapses involved in the manifestation of symptoms typical of 
these conditions, while considering trauma exposure as a contributing factor, could prove crucial to the understanding of anxiety disorders. Methods. Male adult Sprague-Dawley rats were subjected to maternal and/or restraint stress and used to mimic the presence of major life events (e.g. childhood trauma) and mild stress in adulthood, respectively. The expression of genes encoding components important for synaptic plasticity was investigated using pathway-based polymerase chin reaction (PCR) array technology. Human homologue susceptibility candidate genes (selected based on aberrant gene expression observed in rats) were characterised in a case-control association study comprising patients with a primary diagnosis of obsessive-compulsive disorder (OCD), panic disorder (PD) or social anxiety disorder (SAD) for which trauma history was known. A tag single nucleotide polymorphism (SNP) approach was used to genotype human homologues; alternatively targeted nextgeneration sequencing (tNGS) was employed. Genotyping data was statistically assessed in conjunction with trauma history to test for gene-environment interaction.

Results. Several genes (Bdnf, Mmp9, Arc, Ntf4, Egr2, Egr4 and GRM2) were identified as aberrantly expressed in rats with anxiety-like behaviours v. controls. tNGS of ARC, NTF4, EGR2, EGR4 and GRM2 yielded 400 SNP Indel variations, of which 42 were unique to only either patients or controls. Only 4 of these were characterised on dbSNP.

Conclusion. Differentially expressed genes in rats with a trauma history and anxiety-like behaviours can point to candidate susceptibility genes for anxiety disorders in humans, and significantly enhance understanding for the molecular basis of anxiety disorders. The novel variation identified within this study will be genotyped in the remainder of our cohort to assess for association, in conjunction with trauma data. This will provide proof-of-concept for use of this animal model for novel candidacy identification and elucidate any gene-environment interaction that may be occurring in relation to anxiety disorders.

\section{Higher visual functioning \\ A Moodley \\ Grey's Hospital, Pietermaritzburg, South Africa \\ anand.moodley@kznhealth.gov.za}

It's easy to see but seeing is not easy. The brain's interpretation of what we see is complex and not fully understood. One third of the brain is involved in visual perception and visual processing. The primary visual cortex is not the final destination of visual input from the eyes. Supposedly, 2 functionally independent streams (ventral and dorsal) that emerge from the striate cortex account for most of what we perceive. Pathology in these streams present with fascinating clinical syndromes. This talk will expound on some of these syndromes with illustrative cases presented to, hopefully, unravel their intrigue.

The effects of prenatal methylmercury exposure on trace element and antioxidant levels in rat offspring following 6-hydroxydopamine-induced neuronal insult Z M Moosa, ${ }^{*}$ W M U Daniels, M V Mabandla
Discipline of Human Physiology, School of Laboratory Medicine and Medical Sciences, College of Health Sciences, University of

KwaZulu-Natal, Durban, South Africa

*205504378@stu.ukzn.ac.za

Background. Methylmercury $(\mathrm{MeHg})$ is a metal toxin found commonly in the environment. Studies have shown severe neurotoxic effects of $\mathrm{MeHg}$ poisoning, especially during pregnancy, where it crosses the placenta and the blood/brain barrier leading to neurodevelopmental deficits in the offspring. These deficits may predispose offspring to neurodegenerative diseases, with subsequent injury during adulthood.

Methods. In this study we investigated the effects of prenatal methylmercury exposure $(2.5 \mathrm{mg} / \mathrm{l}$ in drinking water from GND 1) on the trace element status in the brain of adolescent offspring at postnatal day (PND) 28. Total antioxidant capacity (TAC) was also measured in blood plasma. The neurotoxin 6-hydroydopamine (6-OHDA) was administered at PND 60 as a model of neuronal insult which was verified by behavioural analysis. Trace element and TAC levels were compared before and after 6-OHDA exposure.

Results. Prenatal MeHg treatment showed significantly higher concentrations of zinc, copper, manganese and selenium in the brain of offspring at PND $28(p<0.05)$. There were also lower brain iron levels in MeHg-exposed adolescent offspring $(p<0.05)$. Following 6-OHDA exposure copper, zinc, and manganese levels were decreased in control and MeHg-exposed animals, but $\mathrm{MeHg}$ animals had significantly higher concentrations than controls $(p<0.05)$. Contrastingly, iron levels in control animals were decreased and significantly lower than $\mathrm{MeHg}$-exposed offspring $(p<0.05)$. Total antioxidant capacity was not significantly different in $\mathrm{MeHg}$ and control groups prior to lesion. However, following 6-OHDA administration, MeHg-exposed animals had a significantly lower total antioxidant capacity than that of controls $(p<0.05)$.

Conclusion. Prenatal MeHg exposure results in trace element dyshomeostasis in the brain of offspring and reduces total antioxidant capacity. This may reflect a mechanism by which methylmercury exerts its neurotoxicity and may predispose offspring for further neurological insults during adulthood.

\section{Paediatric neuropsychiatric movement disorders L Mubaiwa \\ Department of Paediatric Neurology, University of KwaZulu-Natal, \\ Durban, South Africa \\ mubaiwal1@ukzn.ac.za}

Movement disorders are a common manifestation of an underlying paediatric neuropsychiatry disorder. The prevalence and aetiology of paediatric neuropsychiatry movement disorders in resource-poor settings needs to be explored. Identification of the underlying medical disorder can be difficult in a busy paediatric medical and psychiatric wards. Movement disorders are classified as being hyperkinetic or hypokinetic. Hyperkinetic movement disorders such as chorea, ballismus and tremors are often caused by damage to the basal ganglia 
complex in the setting of general medical disorders. Stereotypic movement disorders are typical in Rett syndrome, tic disorders and Tourette's syndrome. Rare metabolic disorders, Huntington's disease and paraneoplastic syndromes also present with neuropsychiatric movement disorders accompanied by behavioural and personality impairment. The paediatric psychiatrist is often consulted to manage the overt disruptive neuropsychiatric aspects accompanying movement disorders. It is paramount that the underlying medical disorder should be identified, along with administering symptomatic management. This presentation will highlight index cases and be a literature review that will focus on an approach to enable pattern recognition of commonly encountered neuropsychiatric movement disorders in the paediatric medical ward.

\author{
The South African national female offenders study \\ M Nagdee, ${ }^{1 \star} \mathrm{L}$ Artz,${ }^{2} \mathrm{C}$ de Clercq, ${ }^{3,4} \mathrm{P}$ de Wet, ${ }^{5} \mathrm{H}$ Erlacher, ${ }^{1}$ \\ S Kaliski, ${ }^{3,4}$ C Kotze, ${ }^{5}$ L Kowalski, ${ }^{1}$ G Lippi, ${ }^{5}$ J Naidoo, ${ }^{6}$ S Naidoo, ${ }^{6}$ \\ J Pretorius, ${ }^{7}$ M Roffey, ${ }^{3,4}$ F Sokudela, ${ }^{5}$ U Subramaney ${ }^{8}$ \\ ${ }^{1}$ Fort England Hospital, Department of Psychiatry, Walter Sisulu \\ University, Grahamstown, South Africa \\ ${ }^{2}$ Gender, Health \& Justice Research Unit, University of Cape Town, \\ South Africa \\ ${ }^{3}$ Valkenberg Hospital, Department of Psychiatry, University of Cape \\ Town, South Africa \\ ${ }^{4}$ Lentegeur Hospitals, Department of Psychiatry, University of Cape \\ Town, South Africa \\ ${ }^{5}$ Weskoppies Hospital, Department of Psychiatry, University of \\ Pretoria, South Africa \\ ${ }^{6}$ Fort Napier Hospital, Department of Psychiatry, University of \\ Kwazulu-Natal, Durban, South Africa \\ ${ }^{7}$ Free State Psychiatric Complex, Department of Psychiatry, \\ University of Free State, South Africa \\ ${ }^{8}$ Sterkfontein Hospital, Department of Psychiatry, University of the \\ Witwatersrand, Johannesburg, South Africa \\ *drmonagdee@gmail.com
}

Background. Several studies have examined sociodemographic and psycholegal factors associated with offending behaviour. In this context, female offenders have received very little attention in comparison with their male counterparts.

Objective. Because of the differences in the nature and quality of offending behavior between males and females, and the scarce literature on female offenders in South Africa, this research aims to explore the sociodemographic, referral, clinical and forensic characteristics of accused women who are referred for observation under the Criminal Procedure Act (CPA), and to compare their profile to that of their male counterparts. The current study seeks to understand specific pathways to offending behaviour, its associated psycholegal factors, and the broader sociocultural context of this vulnerable group of South African women.

Methods. This is a national, multicentre and multiphased study. An initial retrospective, descriptive study of the clinical records of all accused women referred by the courts to large South African forensic mental health units (in the Eastern Cape, Free State, Gauteng, Kwazulu-Natal and Western Cape provinces) for observation under the Criminal Procedure Act (CPA) between 1998 and 2010 is being conducted. The second phase of this study will collect the same retrospective, descriptive data for a randomly selected control group of accused males referred to the same South African forensic mental health units for observation during the study period.

Results. Preliminary results of a pilot study on the demographic, clinical and forensic profiles of accused women referred for observation to Fort England Hospital in the Eastern Cape will be presented. Outcome measures include sociodemographic characteristics, offence profile, psychiatric diagnosis, fitness to stand trial, and criminal capacity.

Conclusion. It is anticipated that the outcome of the study will generate hypotheses regarding South African female offending behaviour and associated psycholegal factors, and will form the basis for future prospective studies in this population. Analysis of such data will assist in devising possible strategies for reduction or prevention of offending behaviour in this population.

\section{Neurobiological consequences of child abuse C Nemeroff}

Department of Psychiatry and Behavioral Sciences, Leonard M Miller School of Medicine, University of Miami, Miami, Florida, USA

cnemeroff@med.miami.edu

Genetic, brain imaging and neurotransmitter studies have revealed the long-term consequences of child abuse and neglect. These changes increase vulnerability to mood and anxiety disorders in adulthood. Exposure to trauma during childhood increases the risk of certain psychiatric disorders beyond the risk associated with adult violence exposure. We have demonstrated a number of long-term neurobiological consequences of child abuse and neglect, including structural and functional brain imaging changes, and neuroendocrine and immune alterations. In particular, alterations in the hypothalamic-pituitary-adrenal (HPA) axis, a major mediator of the stress response, contribute to the long-standing effects of early life trauma. However, not all exposed individuals demonstrate altered HPA axis physiology, suggesting that genetic variations influence the psychiatric consequences of trauma exposure. Variants in the gene encoding the CRF R1 receptor, FKBP5, PAC1 and others interact with adverse early environmental factors to predict risk for stress-related psychiatric disorders. These studies suggest molecular targets for new drug development, biological risk factors, and predictors of treatment response. In addition, the effect of abuse may extend beyond the immediate victim into subsequent generations, as a consequence of epigenetic effects transmitted directly to offspring and/or behavioral changes in affected individuals. Recognition of the biological consequences and transgenerational impact of trauma has critical importance for both treatment research and public health policy.

\footnotetext{
What do Stellenbosch University medical students think about psychiatry - and why should we care? G Nortje, ${ }^{1 \star}$ S Suliman, ${ }^{2}$ K Seed, ${ }^{3}$ G Lydall, ${ }^{4}$ S Seedat ${ }^{1}$ ${ }^{1}$ South African Research Chair in PTSD, Department of Psychiatry, Stellenbosch University, Cape Town, South Africa
} 


\author{
${ }^{2}$ Medical Research Council Anxiety Disorders Unit, Department of \\ Psychiatry, Stellenbosch University, Cape Town, South Africa \\ ${ }^{3}$ Tavistock \& Portman NHS Trust, London, UK \\ ${ }^{4}$ University College London, London, UK \\ *g.nortje@gmail.com
}

Mental illnesses are a major cause of morbidity and mortality worldwide, with increasing relevance to low- and middle-income countries as communicable diseases are slowly tamed. However, there remains a shortage of psychiatrists worldwide, especially in developing countries, which can least tolerate the loss of productivity due to mental illness. Unfortunately, psychiatry is routinely ranked by medical students as one of the least appealing specialties and recruitment into psychiatry suffers as a result. Many studies have examined the correlates of poor student attitudes towards psychiatry, with the hope of identifying both positive and negative factors influencing career choice in psychiatry. However, previous studies have mostly been small, local and only examined a few potential correlates. Stellenbosch University recently took part in the International Study of Students' Career Choice in Psychiatry, the first international large well-powered cross-sectional study. Final-year medical students from 17 different countries were invited to take part in a detailed online survey. In this presentation I will share results from the South African branch of the study, and compare these to results from other countries. I will also outline challenges facing poorer developing countries in recruiting psychiatrists.

\section{Neurological soft skins in Nigerian Africans with first episode schizophrenia: Factor structure and clinical correlates}

\section{A Ojagbemi ${ }^{*}$ O Esan, O Gureje, R Emsley}

College of Medicine, University of Ibadan, Nigeria

*drakinjagbemi@yahoo.com

Background. There is still a paucity of information about the structure of neurological soft signs in African patients with schizophrenia.

Method. A total of 84 patients with first episode Diagnostic and Statistical Manual of Mental Disorders (DSM-IV) schizophrenia or schizophreniform disorder were consecutively recruited. Patients' clinical features were assessed with the Positive and Negative Syndrome Scale, Clinical Global Impression, Calvary Depression Scale for Schizophrenia, and the Premorbid Adjustment Scale. Neurological assessment was based on the 26-item Neurological Evaluation Scale. An exploratory factor analysis of the items on the scale was conducted, and the relationships of the derived factors with the clinical characteristics of schizophrenia were determined.

Results. Neurological soft signs were loaded into a 4-factor structure: perceptual and motor sequencing (audio-visual integration, fist-edge palm, rhythm tapping, extinction, and right-left confusion), eye movements (synkinesis, convergence, and gaze impersistence), motor co-ordination and graphaesthesia (tandem walk, adventitious flow, and graphaesthesia), as well as stegreognosis. These signs significantly correlated with more severe negative and disorganised psychopathology (negative symptoms $r=0.0 .456, p<0.01$; disorganisation $r=0.559, p<0.01$; hostility $r=0.228, p<0.05$; and clinical global impression of severity $r=0.566, p<0.01$ ).

Conclusion. Neurological soft signs in this sample loaded into a 4-factor structure with important overlap with previous empirically derived factors. A preponderance of the signs was associated with a more severe psychopathology, especially with features of negative and disorganisation sympomatology.

\section{Should psychiatric patients know their MTHFR status? \\ E Peter}

Psychiatrist in Private Practice

peterem@mweb.co.za

5,10-methylenetetrahydrofolate reductase (MTHFR) enzyme (OMIM $\# 607093)$ is the key enzyme in the essential vitamin B9, folate metabolism pathway. It is required for the fourth-step transformation of dietary folate to L-methylfolate, which the brain requires for methylation, the process of DNA repair. L-methylfolate is the cofactor of tetrahydrobiopterin (BH4) which builds neurotransmitters. Polymorphisms of C677T MTHFR, C/T and T/T are common and reduce the enzymatic activity to $71 \%$ and $34 \%$, respectively. Many drugs such as lamotrogine and other anticonvulsants may further reduce the absorption of folate. L-methylfolate is being used as an augmentation strategy for treatment resistant depression. This presentation will show how MTHFR T/T has been found in a number of severe bipolar families and augmentation may play a role in improving symptoms. Testing MTHFR T/T status allows for compensation by a US Food and Drug Administration Prescribed Medical Food. Should psychiatric patients know their MTHFR status? Definitely.

\section{Clinical and functional outcome of treatment refractory first-episode schizophrenia}

L Phahladira, ${ }^{*}$ R Emsley, L Asmal, B Chiliza

Department of Psychiatry, Stellenbosch University, Cape Town,

South Africa

*lebogang.phahladira@gmail.com

Background. Most patients who experience a first episode of psychosis (FEP) respond to antipsychotic treatment, with up to $80 \%$ responding within the first year. Patients with ongoing psychotic symptoms are more likely to have a poor outcome and are less likely to benefit from rehabilitation efforts. Treatment-resistant schizophrenia is not a discrete entity; however, there are several predictors of poor outcome that have emerged from follow-up studies of schizophrenia patients.

Objective. This exploratory study aimed to describe the trajectory and outcome of treatment refractoriness in FEP.

Method. In a prospective, longitudinal study of first-episode schizophrenia within the greater Cape Town area, 12 of the 130 participants failed to adequately respond to standardized treatment with flupenthixol decanoate and were classified as treatment refractory. We conducted a cross-sectional descriptive assessment of these patients $(n=12)$ using 
the Positive and Negative Symptoms Scale for Schizophrenia, the Clinical Global Impression, Social and Occupational Functioning Assessment, the World Health Organization Quality of Life Scale and the Neurological Evaluation Scale. The current treatment with antipsychotics and concomitant medications was recorded.

Results. In our sample, we identified demographic, clinical and functional factors associated with treatment refractoriness in schizophrenia. Clinical, functional and treatment outcome in these patients will be described. The limitations of the study are a small sample size and cross-sectional observation.

Conclusion. The evolution of treatment refractoriness in schizophrenia is poorly understood. While some patients are nonresponsive to antipsychotic treatment from the outset, others appear to respond initially, but after experiencing a relapse fail to improve to their previous levels.

\section{Bioethics by case discussion W Pienaar \\ Department of Psychiatry, Faculty of Medicine and Health Sciences, Tygerberg Campus, Stellenbosch University, Cape Town, South Africa wppien@sun.ac.za}

In the discipline of psychiatry, clinicians are challenged with clients made vulnerable by psychiatric illness, and who may be partially competent or incompetent to make a reasonable decision in their own best interest. The conflict between respect for client autonomy versus beneficence often creates ethical dilemmas for the clinician. By examining different case scenarios, the tools of moral deliberation will be discussed. Moral theories of Western philosophy will support our arguments.

\section{Reviewing our social contract pertaining to psychiatric research in children, research in developing countries and distributive justice in pharmacy \\ W Pienaar \\ Department of Psychiatry, Faculty of Medicine and Health Sciences, Tygerberg Campus, Stellenbosch University, Cape Town, South Africa \\ wppien@sun.ac.za}

We are under a social contract. By living and practising our profession under this social contract, we have given up some of our human rights, have taken up responsibilities and are accountable for services we provide in the field of psychiatry. The bioethical questions and complexities of research on psychiatric disorders in childhood are complex. Psychiatric research done in developing countries by researchers from first world countries are riddled with bioethical questions. Distributive justice in the field of psychopharmacology and the availability of expensive psychotropic drugs are a huge challenge. Do we fulfill our social contract, our duty, our responsibility as best we can? Are we not stuck in old paradigms? Arguments will be presented that indicate that we can make it better!

\section{The performance of the MMSE in a heterogeneous elderly} South African population

$\underline{\text { S Ramlall }},{ }^{1 *}$ J Chipps, $^{2}$ A I Bhigjee, ${ }^{3}$ B J Pillay ${ }^{4}$

${ }^{1}$ Department of Psychiatry, College of Health Sciences, University of KwaZulu-Natal, Durban, South Africa

${ }^{2}$ School of Nursing and Public Health, College of Health Sciences, University of KwaZulu-Natal, Durban, South Africa

${ }^{3}$ Department of Neurology, College of Health Sciences, University of KwaZulu-Natal, Durban, South Africa

${ }^{4}$ Department of Behavioural Medicine, College of Health Sciences, University of KwaZulu-Natal, Durban, South Africa

${ }^{*}$ ramlalls4@ukzn.ac.za

Background. The Mini-Mental State Examination (MMSE) is the most researched cognitive screening tool in use worldwide, despite the emergence of many newer tools recently. Its limitations pertain to the influence of demographic and social variables on scores obtained. Objective. We evaluated the performance of the MMSE on a local elderly population.

Methods. A cross-sectional study was conducted among the residents of a group of residential homes for the elderly in Durban. The MMSE was administered to a convenience sample of elderly residents, together with an informant questionnaire, the geriatric depression scale (GDS) and a subjective memory scale. Subsamples of participants with screen-positive and screen-negative scores were diagnosed clinically with either dementia or mild cognitive impairment (MCI).

Results. Lower MMSE scores were significantly associated with increased age groups $(\kappa=9.6, p=0.023)$, lower education groups $(\kappa=12.5, p=0.002)$ and race $\kappa=25.3, p<0.001)$.For every one additional year of education, participants were 0.71 times less likely to screen positive on the MMSE. Differential item functioning on various components of the MMSE was demonstrated due to the effects of education, race and gender. There was significant differential performance between the recommended and alternate attention/ concentration items $(p<0.001)$ with the alternate item favouring better performance. At recommended cut-off scores, the MMSE had significant discriminant validity for controls, dementia and mild cognitive impairment $(p=0.01)$. It had an AUC of 0.77 with a sensitivity of $63.6 \%$ and a specificity of $76 \%$. Increasing the cut-off to $<24$ will improve its sensitivity to $81.8 \%$.

Conclusion. The influence of race, age and education on MMSE scores highlights the need for the MMSE to be adapted for local use. The alternate item for attention/concentration should be preferred.

\section{Biological basis of addiction (alcohol and drug addiction) $\underline{\text { S Rataemane }}$ \\ Medical University of South Africa, University of Limpopo, \\ Turfloop, South Africa \\ srataema@gmail.com}

Addiction to drugs still presents a challenge in clinical practice. A number of theories have been postulated to explain the high relapse 
rate in addiction to alcohol and drugs despite optimal intervention with appropriate pharmacological and psychosocial interventions. The brain reward hypothesis is one such theory and it is associated with specific structures in the brain that are active during addiction stages. This presentation will discuss the role of social learning in development of addictions and relevant psychosocial interventions to reverse the process. Biological underpinnings of addiction will also be discussed, with reference to structures such as the amygdala, anterior cingulate and orbitofrontal glutamatergic projections to the nucleus accumbens.

Volumetric brain changes in prenatal methamphetamineexposed children compared with healthy unexposed controls A Roos, ${ }^{1 *}$ K Donald, ${ }^{2}$ G Jones, ${ }^{1}$ DJ Stein ${ }^{1,3}$

${ }^{1}$ Medical Research Council Unit on Anxiety \& Stress Disorders, Stellenbosch University, Cape Town, South Africa

${ }^{2}$ Division of Developmental Paediatrics, University of Cape Town, South Africa

${ }^{3}$ Department of Psychiatry and Mental Health, University of Cape Town, South Africa

*aroos@sun.ac.za

Background. Methamphetamine (MA) use in South Africa and globally has increased markedly in recent years. Its effect on brain structure in prenatally exposed children is understudied.

Objective. To investigate the potential changes to brain structure of children with prenatal MA exposure, compared to unexposed controls, as measured by magnetic resonance imaging.

Methods. Ten patients ( 5 males, 5 females) known to have had MA exposure during pregnancy and 19 healthy matched controls (8 males, 11 females) were recruited to the study. Groups were matched for age and socioeconomic background. The children underwent 3T structural imaging to investigate brain volumes. Data was analysed for volume size of structures using Freesurfer and compared by group using one-way ANOVA in Statistica 11.

Results. Between group differences were found, with significantly greater volumes in both left $(F(1,27)=11.22, p=0.002)$ and right putamen $(F(1,27)=4.79, p=0.040)$ in MA exposed children compared with unexposed controls. In addition, the left pallidal $(F(1,27)=5.19$, $p=0.031$ ) volume was increased in the MA-exposed group.

Conclusion. The dopamine-rich striatal structures are recognised as being vulnerable to the neurotoxic effects of methamphetamines. The findings of this study further implicate this region as being a key site of the long-term neurobiological effects of MA on the developing brain. The results described add significant findings to an emerging, but small body of literature on this important subject.

Single voxel proton magnetic resonance spectroscopy of the amygdala in social anxiety disorder in the context of early developmental trauma

D Rosenstein, ${ }^{1 *}$ A Hess, ${ }^{2}$ S Seedat, ${ }^{1}$ E Meintjies ${ }^{2}$

${ }^{1}$ South African Research Chairs Initiative in Post-Traumatic Stress

Disorder, Department of Psychiatry, Faculty of Health Sciences,

Tygerberg Campus, Stellenbosch University, Cape Town, South Africa

${ }^{2}$ Biomedical Engineering, University of Cape Town, South Africa
${ }^{3}$ Institute of Biomedical Engineering, University of Oxford, United Kingdom

*rosensteind@gmail.com

Background. Phosphocreatine (PCr) is a major neurometabolite involved in nervous system metabolism, with dysregulation suggesting high levels of emotional distress. Individuals with anxiety disorders demonstrate alterations in the neurometabolism of the limbic system, specifically the amygdaloid complex. This has also specifically been demonstrated in social anxiety disorder (SAD). The early contributions of early developmental trauma have been hypothesised to play a significant role in the pathophysiology of anxiety disorders.

Objective. We used single voxel proton magnetic resonance spectroscopy ( ${ }^{1} \mathrm{H}$-MRS) to compare the neurometabolite profiles of the left amygdala in individuals with SAD $(n=20)$ with early developmental trauma (EDT) compared with individuals with SAD without EDT $(n=22)$ and healthy controls $(n=21)$. (The mean age of the participants was 35.5 years.)

Methods. Subjects were screened with the Mini International Neuropsychiatric Interview (MINI), Liebowitz Social Anxiety Scale (LSAS) and Childhood Trauma Questionnaire (CTQ). Scanning was carried out in a 3T Siemens Allegra Scanner. ${ }^{1} \mathrm{H}-\mathrm{MRS}$ was carried out to elucidate the neurometabolites (NAA, Cr, PCr, Glu, Gln, Glu-Gln and Ins) in the amygdaloid complex and then compare differences across the groups. LCModel was used to analyse neurometabolite profiles with $\mathrm{SAD}=25 \%$.

Results. Significant differences were found in $\operatorname{PCr}(p=0.044)$ and Glu-Gln ( $p=0.015)$ in the left amygdala between the various groups. The greatest change in concentration in Glu-Gln appeared in the SAD without EDT group, followed by the SAD with EDT group. Significant differences were found between the control groups and the SAD without EDT ( $F=-5.64023, p=0.009)$, the SAD with EDT group and the SAD without EDT group $(F=-4.32896, p=0.049)$.

Discussion. Our findings support research that shows alterations in the amygdala in SAD, specifically regarding glutamatergic neurotransmission. Greatest changes appear in the SAD without EDT possibly suggesting distinct pathophysiologies in aetiology of SAD. The differences found in PCr also suggest a novel finding in the dysregulation of amygdala functioning in SAD and SAD with EDT.

\section{Discussion of HDAC inhibitors, with specific reference to sulpiride and its use during breastfeeding \\ J Roux}

Psychiatrist in Private Practice, SIG Cellular and Molecular Psychiatry jproux@worldonline.co.za

Gene activity can be permanently altered in 4 ways: (i) physical rearrangement; (ii) genetic feedback loop; (iii) chromatin modification; (iv) DNA methylation. The histones in heterochromatin are generally unacetylated, whereas those in areas containing active genes are acetylated. Activity depends on the balance between the activities of two enzyme types, the histone acetyl transferases (HATs) and histone deacetylases (HDACs). HDAC inhibitors are widely tested as pharmacological agents to block the activity of HDACs and thus 
enhance the activity of HATs. HDAC inhibitors are essentially gene activators. HDAC inhibitors are emerging as a new class of potential anti-cancer drugs and have been shown to induce differentiation, cell cycle arrest and apoptosis and to inhibit migration, invasion and angiogenesis in many cancer cell-lines. In addition, these compounds inhibit tumour growth. In recent years, an increasing number of structurally divers HDAC inhibitors have been identified. In October 2006, the US Food and Drug Administration approved the first HDAC inhibitor, Virinostat, to treat the rare circulating tumour cell cancer (CTCC). At least 12 different HDAC inhibitors are currently in clinical trials as monotherapy or in combination to treat various types of cancer. Among benzamide HDAC inhibitors, both MS-275 and CI-994, are currently in clinical trials for the treatment of cancer. Sulpiride is also a benzamide and has been shown to activate brain DNA methylation for reelin and GAD67 promoters, and to increase $\mathrm{H} 3-\mathrm{K}$ acetylation at the reelin promoter. We conducted a survey exploring the off-label prescription of sulpiride to breastfeeding mothers by general practitioners. Our results showed that it is still widely practised, that more than $80 \%$ of respondents admit to using sulpiride during breastfeeding and that $62 \%$ feel it is completely safe to do so. If sulpiride proves to have the same HDAC inhibiting properties as extremely cytotoxic and potent anti-cancer treatments, these practices might be harmful to the future neurodevelopment of the breastfeeding infant.

\section{Prevalence and clinical correlates of police contact prior to a first diagnosis of schizophrenia \\ C Schumann, ${ }^{*}$ L Asmal, K Cloete, B Chiliza, R Emsley}

Department of Psychiatry, Faculty of Medicine and Health Sciences,

Stellenbosch University, Cape Town, South Africa

*schucorrie@yahoo.com

Background. While a positive association between criminal behaviour and schizophrenia does exist, this relationship is modest compared with that between criminal behaviour and substance abuse or sociodemographic variables. It is a common misconception that most people suffering from schizophrenia engage in criminal behavior, particulary violent crime, and there is evidence that police services may be susceptible to these misconceptions.

Objective. This study explored the association between first-episode schizophrenia and police contact in the South African context.

Methods. The prevalence and type of previous police-contact in 110 patients with first-episode schizophrenia and 65 healthy, matched controls were compared. Patients with first-episode schizophrenia with a history of police-contact were compared with those without, in terms of sociodemographic and clinical characteristics. Policecontact during the period of untreated psychosis (DUP) and period of untreated illness (DUI) was ascertained.

Results. Patients had significantly more police contact than controls $(p=0.002)$, despite the higher prevalence of conduct symptoms and methamphetamine abuse in controls. However, patients were not more likely to be incarcerated or arrested than controls. In the patient group police contact was associated with male sex ( $p=0.0007)$, shorter education $(p=0.01)$, substance abuse $(p=0.0007)$, methamphetamine abuse $(p=0.02)$ and a history of $\geq 2$ conduct symptoms $(p=0.02)$.
Patients with police contact had more positive $(p=0.0009)$, general $(p=0.02)$ and total symptoms of psychosis $(p=0.01)$, lower social and occupational functioning $(p=0.02)$ and poorer physical health $(p=0.03)$. Logistic regression analysis showed positive psychotic symptoms to be the strongest independent predictor of police contact in patients. Police contact occurred during DUP in 38\%, and from the onset of illness in $70 \%$ of patients.

Conclusion. Police contact is more common in first-episode psychosis (FEP) patients than controls and is associated with untreated positive psychotic symptoms. Early detection and treatment of psychosis may reduce police contact. By identifying FEP patients with psychotic behaviour, the judicial system may potentially play an important role in reducing the duration of untreated psychosis. Further studies are needed to evaluate potential arrest biases and pathways to care that lead to increased police contact.

\section{Are dreams meaningless? \\ M Solms}

Department of Neurology, Groote Schuur Hospital, Cape Town,

South Africa

mark.solms@uct.ac.za

Sigmund Freud proposed that the biological function of dreaming was to protect sleep, and that it did so by fulfilling wishes that arise during sleep in a hallucinatory fashion (in disguised form). The discovery of the brain mechanisms of rapid eye movement (REM) sleep between the 1950s and 1970s cast considerable doubt on this hypothesis: REM sleep occurs automatically in 90-minute cycles and it is generated by a 'mindless' part of the brainstem. This lecture will present findings which show that dreaming is not in fact generated by the part of the brain that generates REM sleep; it does not occur automatically and is generated by a part of the brain that is deeply implicated in 'fulfilling wishes'. Recent findings will also be presented from an ongoing study which is directly testing the hypothesis that dreams protect sleep.

\section{The conscious id \\ M Solms}

Department of Neurology, Groote Schuur Hospital, Cape Town, South Africa

mark.solms@uct.ac.za

Sigmund Freud's proposal that many (if not most) mental functions operate unconsciously is now widely accepted. Like Freud, most contemporary scientists associate consciousness with the brain mechanisms for perceptual awareness, and they associate unconscious mental functions with 'lower' mechanisms localised deeper within the brain. This presentation will argue - on the basis of compelling neuropsychological evidence - that the relationship between the brain mechanisms for conscious and unconscious cognition is just the reverse: consciousness is a far more primitive and basic function than the unconscious mental capacities that are now so topical in cognitive neuroscience. Some implications for psychiatry and for medical ethics will be discussed. 
Depression and resilience in $\mathrm{HIV}$-infected women with early life stress: Does trauma play a mediating role?

$\underline{\mathrm{G} \text { Spies}},{ }^{1 \star}$ S Seedat ${ }^{1,2}$

${ }^{1}$ South African Research Chairs Initiative (SARChI), PTSD program, Department of Psychiatry, Stellenbosch University, Cape Town, South Africa

${ }^{2}$ Medical Research Council Unit on Anxiety and Stress Disorders, Department of Psychiatry, Stellenbosch University, Cape Town, South Africa

*ggiocos@sun.ac.za

Background. Common mental disorders such as depression and anxiety are highly prevalent in HIV-infected individuals. A broad range of adult psychopathology has been reported in studies of HIVinfected individuals with a history of childhood trauma. However, there is relatively little awareness about resilience and its relationship to these disorders. To our knowledge, there are no studies investigating the relationship between depressive symptomatology and resilience in a sample of trauma exposed HIV-infected individuals.

Objectives. To assess the relationship between depressive symptomatology and resilience among HIV-infected women and to investigate whether childhood trauma, stressful life events, or posttraumatic stress disorder (PTSD) symptomatology mediated this relationship. Data were collected from a convenience sample of 95 HIV-infected women in peri-urban communities in the Western Cape Province, South Africa. All the women had exposure to childhood trauma as determined by the Childhood Trauma Questionnaire (CTQ). Participants completed self-report instruments assessing depressive symptomatology, stressful life events, PTSD symptoms and resilience.

Results. There was a significant negative correlation between depressive symptomatology and resilience $(p<0.01)$. Partial Least Squares (PLS) analysis revealed a significant direct effect between depression and resilience. The Sobel test for mediation revealed no significant results for childhood trauma or stressful life events $(p>0.05)$. However, PTSD symptomatology significantly mediated the relationship between depression and resilience in these traumaexposed women living with HIV. PLS analysis confirmed these results. Conclusion. In the present study, higher levels of resilience resulted in lower levels of self-reported depression. This suggests that among this sample, resilience may act as protective factor against the development of clinical depression. The results also demonstrated that self-reported PTSD symptoms mediated this relationship. Although there was a direct effect of resilience on depression, the presence of PTSD symptoms, which are highly prevalent in HIV-infected and traumaexposed individuals and often comorbid with depression, may further explain and account for this relationship.

\section{State of affairs analysis for forensic psychiatry in SA U Subramaney* \\ School of Clinical Medicine, Faculty of Health Sciences, University of the Witwatersrand, Johannesburg, South Africa \\ *ugasvaree.subramaney@wits.ac.za}

Background. Forensic psychiatric patients represent a very marginalised group that psychiatrists have to deal with.The Mental Health Care
Act (MCHA) of 2002 has not been wholly successful in its quest to address disparities in mental health with regard to the management of forensic state patients.

Objective. To uncover the current challenges with regard to the management, observation and follow-up in terms of the management of the state patients in terms of section 42 of the MCHA.

Methods. This will be in the form of a review of the forensic special interest group (SIG) and state-employed special interest group (SESIG) situation across all provinces in South Africa. References will be made to the SESIG weekend deliberations as well as the deliberations of the interdepartmental meeting held at OR Tambo in April this year. The current 'state of affairs' analysis for regions will include variables such as: population; gender; ethnicity; public/private. The referral system will be reviewed based on designated units, i.e. number and type of beds. Facilities will be audited in terms of the National Mental Health Action Plan: new inpatient units required; state of existing units, community residential facilities; human resources (posts available and filled for interns, medical officer's registrars, and psychiatrists). The minimum dataset will include average length of stay, bed occupancy, admissions, OPD and consultation/liaison. Clinical data will include diagnoses (ICD) and treatment, and personalised recovery plans.

Results. Major challenges still exist in forensic mental health. Policies, infrastructure, protocols of management, research and communication issues were found to be major factors that need to be addressed to bring about substantial change in these services.

Conclusions. Strategic planning with action plans, indicators and time frames needs to be implemented so that forensic psychiatry in South Africa can be uplifted on all fronts.

\section{Escitalopram in the prevention of post-traumatic stress disorder: A pilot randomised controlled trial $\underline{\text { S Suliman }},{ }^{1 *}$ S Seedat, ${ }^{1,2}$ J Pingo, ${ }^{1}$ T Sutherland, ${ }^{1}$ \\ J Zohar, ${ }^{3}$ D J Stein ${ }^{1,4}$ \\ ${ }^{1}$ Medical Research Council Anxiety Disorders Unit, Department of Psychiatry, Faculty of Medicine and Health Sciences, University of Stellenbosch, Cape Town, South Africa \\ ${ }^{2}$ Department of Psychiatry, Faculty of Medicine and Health Sciences, University of Stellenbosch, Cape Town, South Africa \\ ${ }^{3}$ Department of Psychiatry, Tel-Aviv University, Israel \\ ${ }^{4}$ Department of Psychiatry and Mental Health, University of Cape \\ Town, South Africa \\ *sharain@sun.ac.za}

Background. A small literature suggests that pharmacotherapy may be useful in the prophylaxis of post-traumatic stress disorder (PTSD) in patients presenting with major trauma. There are relatively little data, however, on the use of selective serotonin reuptake inhibitors (SSRIs) in this context.

Methods. Thirty-one participants presenting immediately after trauma, and meeting diagnostic criteria for full or partial acute stress disorder were randomised to treatment with $10 \mathrm{mg}-20 \mathrm{mg}$ escitalopram v. placebo for 24 weeks. Two participants were excluded due to early drop out, leaving 29 (escitalopram $n=17$, placebo $n=12$ ) for intent to treat analyses. Participants were followed up until 52 weeks, with assessments including the Clinician Administered PTSD Scale (CAPS). A repeated measures analysis of variance was undertaken to 
determine the efficacy of the intervention on the CAPS and a $\chi^{2}$ test to determine if escitalopram was successful in preventing PTSD.

Results. There were no significant differences on the CAPS mean scores (mean reduction -46.4 v. $-33.2 ; F=2.06 ; p=0.16$ ) or with regards to the numbers of participants who developed PTSD $(8.3 \%$ v. $\left.11.8 \% ; \chi^{2}=0.089 ; p=0.765\right)$ in each group. There were also no significant differences in secondary measures including the Clinical Global Impressions Scale, and scales assessing depression, anxiety and disability. Improvements in scores in the entire sample were maintained at the 52-week follow-up. Side-effects were comparable across groups.

Conclusions. These data are consistent with other recent work indicating that the SSRIs may not be efficacious in the prevention of PTSD. Although the data are limited by the small sample size, the possibility of PTSD pharmacotherapeutic prophylaxis remains an important one from conceptual and clinical perspectives.

\section{Epigenetic consequences of adverse early social experiences in primates \\ $\underline{\text { S Suomi }}$ \\ Laboratory of Comparative Ethology, NICHD, NIH, \\ Bethesda, Maryland, USA \\ suomis@lce.nichd.nih.gov}

Adverse early social experiences can have lasting effects on primate bio-behavioural development, especial in the context of subsequent social stress. For example, rhesus monkeys raised without their biological mother (but with access to peers) or by neglectful mothers show relatively normal bio-behavioural development when subsequently maintained in benign social environments, but under socially stressfully circumstances, e.g. social separation, they typically exhibit excessive fearfulness and/or aggression, heightened hypothalamic-pituitary-adrenal axis (HPA) activity, and reduced serotonin metabolism into adulthood. Moreover, they differ from monkeys, which did not experience such early social adversity in both brain structure and function. Some of these characteristics appear to be transmitted to their offspring via non-genetic (most likely epigenetic) mechanisms. Recent technological advances in genomics have made it possible to examine genome-wide expression, and preliminary analyses suggest that such adverse early experiences affect approximately one-fifth of the entire rhesus monkey genome (more than 4400 individual genes), both in the brain and in white blood cells. Many of the behavioural and biological consequences of adverse early social experience are largely reversible following targeted environmental interventions: the question of whether the patterns of gene expression in these monkeys are also reversible is under intense current investigation.

\author{
Risk, resilience, and gene $x$ environment \\ interactions in primates \\ S Suomi \\ Laboratory of Comparative Ethology, NICHD, NIH, Bethesda, \\ Maryland, USA \\ suomis@lce.nichd.nih.gov
}

Recent research with both humans and rhesus monkeys has provided compelling evidence of gene/environment $(\mathrm{GxE})$ interactions throughout development. For example, a specific polymorphism of the serotonin transporter (5-HTT) gene is associated with deficits in infant neurobehavioral functioning, extreme responsiveness to social stressors, poor control of aggression, and low serotonin metabolism during juvenile and adolescent development, as well as excessive alcohol consumption in early adulthood, in monkeys reared with peers but not in monkeys reared by their mother. One interpretation of these findings is that secure attachment relationships somehow confer resiliency to individuals who carry alleles that may otherwise increase their risk for adverse developmental outcomes ('maternal buffering'). Similar patterns of apparent 'buffering' have been demonstrated for $\mathrm{GxE}$ interactions involving several other genes with functionally equivalent polymorphisms in both humans and rhesus monkeys. Recent research has suggested that much of this 'buffering' may be taking place in the context of early face-to-face interactions between rhesus monkey infants and their mothers. Moreover, the allelic variation seen in these genes in rhesus monkeys and humans, but apparently not in other primate species, may actually contribute to their remarkable adaptability and resilience at the species level.

\section{Biological aspects of anorexia nervosa C Szabo}

Department of Psychiatry, Faculty of Health Sciences, University of the Witwatersrand, Johannesburg, South Africa

christopher.szabo@wits.ac.za

A recent report on the efficacy of subcallosal cingulate deep brain stimulation for the treatment of refractory anorexia nervosa represents a significant milestone. Treatment of anorexia nervosa has traditionally involved psychotherapeutic approaches, with limited utility for pharmacological interventions, and psychosocial aspects (of the bio-psycho-social approach) typically pre-eminent. The possibility of an efficacious biological intervention may herald a new era in the treatment of anorexia nervosa.

\section{Agents used and profiles of non-fatal suicidal behaviour in East London \\ H Uys}

East London Mental Health Unit, Cecilia Makiwane Hospital, East London, South Africa

psyc@sainet.co.za

Background. The incidence of suicidal behaviour in South Africa compared with other countries is high. Organophosphate was found to be the most common single agent ingested in non-fatal suicidal behaviour amongst the youth in a previous study done in Frere Hospital in East London.

Objective. To investigate the most common agents ingested in patients admitted with suicidal behaviour to Cecilia Makiwane Hospital on the outskirts of East London.

Methods. A prospective study of all non-fatal suicide attempts referred to the Mental Health Unit, Cecilia Makiwane Hospital, 
between March 2009 and February 2010 was undertaken. All patients, who attempted suicide by means of overdose or poisoning were included. The agents ingested were studied and classified into single, multiple or unknown. Demographic data included additional factors such as age, gender, race and number of attempts.

Results. During this 1 -year period 180 patients were referred to the Mental Health Unit and 172 were eligible to participate in the study. Of these patients 112 were female (65\%) and 60 male (35\%). Most were young, with 69 aged between 10 and 20 (40\%) and 66 aged between 20 and $30(38 \%)$. Most patients $(n=131)$ had no previous attempt $(75 \%$ first attempt). Only 1 patient was not African. Most patients ingested a single agent (52\%) followed by multiple agents (40\%). The most common single agent ingested was organophosphates followed by rodenticides and combined antiretroviral agents. The most common agents ingested when multiple agents were used in non-fatal suicidal behaviour were paracetamol, ibuprofen and vitamin B complex.

Conclusion. Organophosphate was the single most common agent used in non-fatal suicidal behaviour, concordant with previous research done in East London. The youth remain at high risk. Suicide prevention should include a multifaceted approach including means restriction.

\section{The contributions of G-protein coupled receptor signalling to opioid dependence \\ Lvan Tonder}

Department of Pharmacology, School of Medicine, Faculty of Health Sciences, University of Pretoria, Pretoria, South Africa

japie.vantonder@up.ac.za

Opioid analgesics offer superior efficacy, but their numerous side effects include sedation, respiratory suppression, tolerance, physical dependence and addiction. Four distinct opioid receptors (ORs) are known, the mu ( $\mu$-OR), delta $(\delta-\mathrm{OR})$, kappa ( $\kappa-\mathrm{OR})$, and nociceptin/ orphanin FQ (ORL-1) receptors, all of which are G-protein coupled receptors. Activation, predominantly of $\mu$-ORs, initiates intracellular signalling cascades that produce the desired antinociceptive effects. The degree of OR signalling, and hence analgesic efficacy, is determined not only by the activation of signalling cascades, but also by regulatory mechanisms that control signalling extent and duration. Prolonged or repeated activation of the $\mu$-OR stimulates intrinsic regulatory mechanisms leading to a decrease in OR signalling, which manifests clinically as opioid tolerance and dependence. This is commonly referred to as receptor desensitisation. 'Classical' or homologous receptor desensitisation defines a proximal mechanism that inhibits signalling at the level of the receptor itself. Key components of this mechanism are the GPCR kinase (GRK) and $\beta$-arrestin ( $\beta$-arr) families of proteins. Following receptor activation, ORs are phosphorylated by GRKs, which allows cytosolic $\beta$-arr to bind to the OR-phosphate complex. This prevents further coupling of the OR to its G-protein, eliminating further signalling. Diminished signalling potential translates into increased dosages and frequencies required to maintain the desired response. This process is reversible through receptor resensitisation, during which incapacitated receptors are recycled back to the cell surface. Clinically, this explains why abstinence from opioid therapy allows recuperation of a normal response to opioid analgesia. Heterologous desensitisation involves the signalling cascade components, downstream of the OR.
Normal OR signalling activates secondary messengers, e.g. protein kinases. Protein kinase secondary messengers are also capable of phosphorylating OR receptors, thereby disabling signalling. However, protein kinases can also phosphorylate ORs other than the one from which they originated, causing desensitisation on a cellular level and contributing to the clinical manifestation of opioid dependence.

\section{Emerging trend and innovation in PTSD and OCD J Zohar}

Division of Psychiatry, Chaim Sheba Medical Center,

Tel Hashomer, Israel

joseph.zohar@sheba.health.gov.il

Post-traumatic stress disorder (PTSD): Since PTSD develops after a specific trauma, there may be a window of opportunity - the time from exposure until PTSD develops - to intervene and prevent its development. The challenge is to find out if this is so and what type of early intervention is effective for those at a high risk of developing PTSD. Cortisol - the 'stress hormone' - is a cornerstone in the normal response to traumatic events. An animal model using rats with hyperreactive hypothalamic-pituitary-adrenal axis (HPA) axis (the Fischer strain), or hypo-reactive HPA axis (the Lewis strain), showed that plasticity of the HPA axis is critical for recovery from a traumatic event. Furthermore, normal hyper-secretion of cortisol following exposure to a traumatic event was associated with a reduction in the amplitude of the memory-fear associated with the exposure. In a pilot study, administration of cortisol (100 mg - $140 \mathrm{mg}$ of hydrocortisone intravenously) was associated with significant favorable change in the trajectory of PTSD. Individuals who got injections no later than 6 hours after exposure to trauma were less likely to develop PTSD in 2 weeks, 1 months and 3 months compared with individuals who got placebo. Obessive compulsive disorder (OCD): The prevalence of OCD amongst schizophrenic patients ranges from 10 to $25 \%$ and has a negative effect on prognosis. The high prevalence often leads to the diagnosis of severe OCD as schizophrenia. The changes introduced in the Diagnostic and Statistical Manual (DSM) 5 helps to clarify this challenging diagnostic issue. DSM 5 includes a new insight specifier composed of 3 levels: good or fair insight, poor insight and absent of insight (delusional). The third category 'allows' the diagnosis of OCD even if it appears 'delusional'. It actually suggests that severe (psychoticlike) OCD is still an OCD and not schizophrenia. The diagnostic and treatment implications of those changes will be discussed.

\section{Making the SASOP treatment guidelines operational $\underline{\text { E Allers }}$}

Psychiatrist in Private Practice

kopshop@global.co.za

The South African Society of Psychiatrits (SASOP) produced an evidence-based treatment guideline for 2013. The document does not include all the practical aspects of a practice treatment guideline for the private sector. During a meeting on the weekend of $13-14$ April 2013, the meeting delegated PsychMg to make the treatment guideline operational for the private sector. The presentation aims to describe the process of making the guidelines operational for the private sector. 


\section{POSTER PRESENTATIONS}

Neuropsychological deficits in social anxiety disorder in the context of early developmental trauma

S Bakelaar, ${ }^{\star}$ D Rosenstein, S Seedat

South African Research Chairs Initiative in Post-Traumatic Stress Disorder, Department of Psychiatry, Faculty of Health Sciences, Tygerberg Campus, Stellenbosch University, Cape Town, South Africa *susanbakelaar@hotmail.com

Background. Previous research links early developmental trauma (EDT) to neuropsychological impairments later in life. EDT is frequently reported in individuals with social anxiety disorder (SAD). Neurocognitive aspects of SAD in the context of EDT have not been fully elucidated.

Methods. A neuropsychological test battery assessed cognitive performance in visual and verbal memory, attention/inhibition and executive functioning in individuals with SAD with $(n=25)$ and without $(n=24)$ EDT and in healthy controls (HC) $(n=26)$. Groups were compared on neuropsychological domains. Non-parametric Kruskal-Wallis tests explored group differences on outcomes. Post $h o c$ analyses explored group differences, using non-parametric Mann-Whitney tests with an adjusted significance level of $p=0.0167$ (Bonferroni correction).

Results. Significant differences were found between the HC and SAD with EDT groups only, on the grooved pegboard (dominant and, $p=0.008$; non-dominant hand, $p=0.013$ ); the Wechsler logical memory task (immediate $p=0.009$; and delayed recall $p=0.013$ ); the Stroop colour/word task $(p=0.014)$; and the Hopkins task (immediate $p=0.009$ ); and delayed recall $(p=0.011)$. The HC scored significantly better than the SAD group with EDT. The differences were not accounted for by EDT $(p>0.05)$ or by between-group differences in SAD symptom scores in the SAD groups with and without EDT $(p>0.05)$.

Conclusion. Deficits in immediate and delayed recall of logical memory and verbal learning were found in the SAD group with EDT compared with the HC group, as were deficits in attention/inhibition. This indicates that individuals with a combination of SAD and EDT have significantly higher levels of impairment compared with HCs, suggesting that a combination of SAD and EDT may lead to more pronounced neuropsychological deficits later in life.

Social anxiety disorder in patients with or without early childhood trauma: Relationship to behavioral inhibition and activation and quality of life

$\underline{\text { S Bakelaar }},{ }^{1 *}$ C Bruijnen, ${ }^{2}$ A Sambeth, ${ }^{2}$ S Seedat ${ }^{1}$

${ }^{1}$ Department of Psychiatry, Stellenbosch University, Cape Town, South Africa

${ }^{2}$ Department of Psychology, Maastricht University, Maastricht, Netherlands

*susanbakelaar@hotmail.com

Background. Social Anxiety Disorder (SAD) is the third most common psychiatric disorder in the United States with a grave impact on quality of life (QoL). Studies link early childhood trauma (CHT) to the development of SAD later in life. This study investigated the relationship between SAD, behavioural inhibition and activation systems (BIS and BAS), and QoL in participants with and without exposure to early CHT.

Methods. Demographic and clinical data were collected for 54 participants. According to the Liebowitz Social Anxiety Scale, 42 participants met the criteria for SAD. The Childhood Trauma Questionnaire revealed an exposure to early CHT in 24 participants (SAD positive), and no exposure in 18 participants (SAD negative). BIS and BAS were measured using Carver and White's BIS/BAS Scales and QoL using the Quality of Life Enjoyment and Satisfaction Questionnaire.

Results. This study shows a significant relationship between early $\mathrm{CHT}$ and the later development of SAD, and a positive relationship between the severity of symptoms and the extent to which CHT was experienced. There was a significant difference between the control and SAD groups regarding behavioural inhibition, but not behavioural activation. We conclude that behavioural inhibition is potentially only slightly affected by CHT, or more affected by social anxiety than CHT. Conclusion. Perceived QoL was significantly lower in the SAD populations, and the level of reported $\mathrm{CHT}$ was positively related to perceived QoL. With no significant difference in QoL found between the $\mathrm{SAD}$-positive and $\mathrm{SAD}$-negative groups, we assume only a moderate to small effect of CHT on QoL compared to SAD symptoms on QoL.

\section{Exploring altered affective processing in obsessive- compulsive disorder symptom subtypes \\ E Breet, ${ }^{1 *}$ J Ipser, ${ }^{2}$ D Stein, ${ }^{2}$ C Lochner ${ }^{1}$}

${ }^{1}$ Medical Research Council Unit on Anxiety and Stress Disorders, Department of Psychiatry, Stellenbosch University, South Africa

${ }^{2}$ Department of Psychiatry \& Mental Health, University of Cape Town, South Africa

*elsie@sun.ac.za

Background. Altered affective processing may underlie the clinical manifestation of obsessive compulsive disorder (OCD). While some studies have found deficits in the perception of disgust in OCD, others have failed to replicate this finding, which may be ascribed to the clinical heterogeneity of OCD. Subtyping based on OCD symptomatology may render more homogenous groups and consequently, more consistent data. OCD subtypes have not been considered in studying emotion perception. Therefore this study examined the perception of disgust and other emotions, of different intensities, in OCD symptom subtypes.

Methods. A sample of 20 patients (10 female, 10 male) with a mean age of 35.2 years and a primary diagnosis of OCD without comorbidity were interviewed using the Yale-Brown ObsessiveCompulsive Symptoms Checklist (YBOCS-CL). All patients had moderate to severe OCD. Additionally, a response bias task measured participants' bias when uncertain what facial expression (emotion) is being displayed. Factor analysis of the YBOCS was done to subtype 
the sample according to primary OCD symptom. Groups were then compared in terms of their performance on the response bias task using a mixed model approach.

Results. Preliminary data analyses are underway, and results will be presented at this conference. Given previous data supporting deficits in disgust recognition in OCD patients, and in the contamination/cleaning symptom subtypes specifically, it is expected that our findings will demonstrate bias (higher level of recognising) toward disgust among these subtypes. There may also be differences in perception of the other emotions across symptom subtypes. These findings contribute to the ongoing conversation about emotion perception in OCD.

To investigate the bias toward recognising facial expression of disgust in obsessive compulsive disorder as well as the effect of escitalopram

\section{E Breet, ${ }^{1 *} \mathrm{~J} \mathrm{Ipser}^{2}{ }^{\mathrm{D}}$ Stein, ${ }^{2}$ C Lochner ${ }^{1}$}

${ }^{1}$ Medical Research Council Unit on Anxiety and Stress Disorders, Department of Psychiatry, University of Stellenbosch, South Africa

${ }^{2}$ Department of Psychiatry and Mental Health, University of Cape

Town, South Africa

*elsie@sun.ac.za

Background. Studies have demonstrated that patients with obsessive compulsive disorder (OCD) display impairment in the recognition of facial expressions of disgust. However, the literature on emotion recognition in OCD is inconsistent.

Objective. To examine whether OCD is associated with response bias toward disgust, and if so, whether a single administration of a serotonin reuptake inhibitor (SSRI) escitalopram decreases this bias.

Methods. The sample consisted of 20 patients with a primary diagnosis of OCD and 21 gender- and age-matched healthy controls. One dose of escitalopram (20 mg) or a placebo was administered to both patients and controls 3 hours before the administration of a computerised response bias task on 2 separate assessment days. Participants were presented, for a very brief period, with different facial expression stimuli in varying intensities. Participants were then required to indicate if the emotion displayed sadness, anger, fear or disgust. Some facial expression stimuli were completely neutral but the lack of a neutral option forced patients to select an emotion. This task investigated whether participants' responses to the neutral stimuli displayed bias toward a particular emotion. Data analysis investigated performance of the OCD v. control groups within both conditions (escitalopram v. placebo) separately, with a repeated methods analysis of variance using a mixed model approach. The effects of various demographic variables and severity of symptoms were controlled for.

Results. Preliminary data analyses are currently underway, and results will be presented at this conference. These analyses may contribute to the ongoing conversation on emotion perception in OCD.

\section{A fatal case of nevirapine-induced Stevens-Johnson's syndrome in HIV mania}

A Bronkhorst, ${ }^{1 \star}$ Z Zingela, ${ }^{1}$ W M Qwesha, ${ }^{2}$ B P Magigaba $^{2}$

${ }^{1}$ Department of Psychiatry Port Elizabeth Hospital Complex, Port Elizabeth, South Africa

\author{
${ }^{2}$ Department of Dermatology Port Elizabeth Hospital Complex, Port \\ Elizabeth, South Africa \\ *77alti@gmail.com
}

Background. Mania with psychotic features is one of the most common presenting clusters of psychiatric symptoms in HIVpositive patients, requiring antiretroviral treatment (ART), mood stabilizers and antipsychotics. We report a fatal case of nevirapineinduced Stevens-Johnson syndrome. Holistic management requires that clinicians managing HIV-positive patients with concomitant psychiatric syndromes also have adequate knowledge about ART and the risks of associated side effects.

Method. The patient's mother consented to our write-up of this case report on a 28-year-old HIV-positive male. He was admitted to the acute mental health unit following a first presentation with mania. He was referred from the local general medical hospital with a history of manic and psychotic symptoms. His most recent $\mathrm{CD} 4^{+}$count was 334, taken during the month of admission. He was aware of his HIV status but was not yet on ART. This case report details the patient's presenting symptoms, management, response to treatment and outcome. Incorporated are details of his progress in both the acute psychiatry unit and subsequent management in the intensive care unit, as well as details of the intervention from the dermatology team who managed his serious adverse skin reaction, diagnosed as toxic epidermal necrolysis. Factors contributing to the fatal outcome are also highlighted.

Conclusion. It is imperative to disseminate the details of this case because ( $i$ ) there have been case reports of psychotropic medication inducing severe cutaneous reactions, and (ii) there was a causative agent other than psychotropics identified in this case. This report may alert clinicians to this uncommon but potentially fatal complication.

Association of the COMT G472A (met/met) genotype with lower disability in people diagnosed with multiple sclerosis W Davis ${ }^{1,2 *}$ S J van Rensburg, ${ }^{1,2}$ L Fisher, ${ }^{2,3}$ F J Cronje, ${ }^{1,2}$

D Geiger, ${ }^{2,3}$ M J Kotze ${ }^{2,3}$

${ }^{1}$ Department of Chemical Pathology, Stellenbosch University, Cape Town, South Africa

${ }^{2}$ National Health Laboratory Service, Cape Town, South Africa

${ }^{3}$ Department of Anatomical Pathology, Stellenbosch University, Cape Town, South Africa

*14815524@sun.ac.za

Background. A polymorphism (rs4680, G472A, Val158Met) in exon 4 of the catechol-O-methyltransferase (COMT) gene results in a valine to methionine (Val/Met) amino acid change at codon 158 of membrane-bound COMT (MB-COMT). This results in thermolability, altering protein abundance. The resulting threeto four-fold decrease in enzyme activity reduces noradrenaline catabolism within synapses. The $G$ and $A$ alleles thus produce high- and low-activity enzymes respectively. The G allele frequency is approximately $49 \%$ in whites: $24 \%$ of this population is homozygous for the G/G (Val) high activity genotype, with $27 \%$ homozygous for the A/A (Met) low activity genotype. This lowering of activity and 
resulting reduction of noradrenaline catabolism within synapses is postulated to decrease inflammation occurring in neuroinflamatory diseases. The high activity COMT enzyme could therefore decrease noradrenaline, leading to central nervous system (CNS) inflammation in multiple sclerosis (MS) patients.

Objective. To investigate this COMT polymorphism in relation to disability status in patients with MS.

Methods. MS patients $(n=51)$ were assessed using questionnaires and biochemical tests, including assessment of disability status using the Expanded Disability Status Scale (EDSS). DNA was extracted from all samples for genotyping of functional single nucleotide polymorphisms (SNPs) in the COMT (G472A, rs4680) gene. High throughput realtime polymerase chain reaction (RT-PCR) methodology was applied, following analytical validation against direct sequencing as the gold standard. Statistical analysis was performed to evaluate associations.

Results. The minor low-activity COMT rs4680 (472 G>A) allele was associated with lower disability $(p=0.040)$, after adjusting for gender. Patients with the COMT rs4680 (G472A) A/A (Met/Met) genotype scored on average 1.9 points lower than patients with Val/Met or Val/ Val alleles. This shows a possible protective recessive effect of COMT rs4680 (472 G>A) A/A genotype on EDSS.

\section{Homocysteine levels are associated with the fat mass and obesity associated gene $\mathrm{FTO}$ (intron $1 \mathrm{~T}>\mathrm{A}$ ) polymorphism in MS patients \\ W Davis ${ }^{1,2 *}$ S J van Rensburg, ${ }^{1,2}$ M J Kotze, ${ }^{2,3}$ L Fisher, ${ }^{2,3}$ M Jalali, ${ }^{4}$ \\ F J Cronje, ${ }^{2}$ K Moremi, ${ }^{1,2}$ J Gamieldien, ${ }^{4}$ D Geiger, ${ }^{2,3}$ M Rensburg, ${ }^{1,2}$ \\ R van Toorn, ${ }^{5} \mathrm{M}$ J de Klerk, ${ }^{1,2} \mathrm{G}$ M Hon, ${ }^{6} \mathrm{~T}$ Matsha,${ }^{6}$ S Hassan, ${ }^{6}$ \\ R T Erasmus ${ }^{1,2}$ \\ ${ }^{1}$ Department of Chemical Pathology, Stellenbosch University, Cape Town, South Africa \\ ${ }^{2}$ National Health Laboratory Service, Cape Town, South Africa \\ ${ }^{3}$ Department of Anatomical Pathology, Stellenbosch University, Cape Town, South Africa \\ ${ }^{4}$ Knowledge Integration and Biomarker Discovery, South African National Bioinformatics Institute, University of the Western Cape, South Africa \\ ${ }^{5}$ Paediatric Medicine and Child Health, Stellenbosch University, Cape Town, South Africa; \\ ${ }^{6}$ Department of Bio-Medical Sciences, Faculty of Health and Wellness \\ Science, Cape Peninsula University of Technology, Cape Town, South Africa \\ *14815524@sun.ac.za}

Background. High homocysteine levels have been associated with obesity and multiple sclerosis (MS). Obesity is also a significant risk factor for development of early-onset MS. The fat mass and obesity associated (FTO) gene has not previously been studied in relation to MS and homocysteine, an important marker of dysfunctional methylation. This study evaluated the influence of vascular risk factors, including body mass index (BMI), total cholesterol levels and the FTO rs9939609 (intron 1 T>A) single nucleotide polymorphism (SNP) in relation to homocysteine levels.

Methods. A total of 108 white MS patients and 197 control individuals were genotyped for FTO rs9939609 using real-time polymerase chain reaction (RT-PCR). Homocysteine levels were subsequently measured in a subgroup of 60 patients and 87 controls and correlated with selected vascular risk factors.

Results. A statistically significant association $(p=0.006)$ was observed between homocysteine levels and FTO rs9939609, with a 15\% increase in the presence of each risk-associated A allele. Homocysteine levels were significantly higher in males than females $(p<0.001)$ as expected, with a $15 \%$ increase associated with the A allele after adjustment for gender. Total cholesterol levels $(p=0.049)$ and BMI $(p=0.046)$ correlated significantly with increased homocysteine levels in the age-, gender- and MS-status adjusted group and in age- and MS-adjusted females respectively, with no difference noted between MS patients and controls.

Conclusion. This study provides evidence for FTO rs9939609 as a genetic link between obesity and MS. Increased homocysteine levels, associated with both MS and vascular risk factors in our study population, may identify a new disease pathway in MS mediated by genetic variation in the FTO gene.

Analysis of the COMT $472 \mathrm{G}>\mathrm{A}$ (rs4680) polymorphism in relation to environmental influences as contributing factors in patients with schizophrenia

D de Klerk, ${ }^{1,2 \star}$ S J van Rensburg, ${ }^{1,2}$ R A Emsley, ${ }^{2}$ D Geiger, ${ }^{2,3}$

M Rensburg, ${ }^{1,2}$ R T Erasmus, ${ }^{1,2}$ M J Kotze ${ }^{2,3}$

${ }^{1}$ Department of Chemical Pathology, Stellenbosch University,

Cape Town, South Africa

${ }^{2}$ National Health Laboratory Service, Cape Town, South Africa

${ }^{3}$ Department of Anatomical Pathology, Stellenbosch University,

Cape Town, South Africa

*sjvr@sun.ac.za

Background. Schizophrenia is a multifactorial condition, with both genetic and environmental factors contributing to pathology. Clinical diagnosis considers personal and family medical history, emotional history, current symptoms, and the exclusion of other psychiatric disorders. The gene encoding catecholamine-O-methyl transferase (COMT) is an important candidate for susceptibility or severity of schizophrenia due to its proven effect on the catabolic clearance of dopamine and homocysteine levels.

Methods. Whole blood samples were collected from 34 study participants, including 9 male patients diagnosed with schizophrenia ( 3 black, 3 coloured, 2 white and 1 Indian) and 35 white controls. Urine samples were obtained from patients and a spot test performed to determine possible cannabis use. Homocysteine levels were determined and DNA was extracted from all samples for genotyping of functional single nucleotide polymorphisms (SNPs) in the COMT (G472A, rs4680) gene. High throughput real-time polymerase chain reaction (RT-PCR) was performed, following analytical validation against direct sequencing as the gold standard.

Results. Seven patients diagnosed with schizophrenia (78\%) had homocysteine levels above the relevant reference ranges for age and gender, compared with only $9(36 \%)$ in the control group. Based on the urine spot test, only 2 patients used cannabis. Four schizophrenic patients (44\%) were smokers, compared with only 1 control. The allele frequencies and genotype distribution of COMT G472A 
(rs4680) determined in the control population as part of the assay validation process, were similar to those previously described in the South African population. The COMT G/G genotype (high-activity allele) was associated with the highest homocysteine levels in the patient population, compared with the lowest levels in the control group.

Conclusion. Our findings emphasise the importance of taking both genetic and environmental factors into account for risk management. COMT genotyping may facilitate the clinical management of patients with schizophrenia through early detection of hyper-responders to environmental influences on dopamine levels. Ultimately, different pieces of information could be translated into a single estimate of risk or intervention plan aimed at prevention of cumulative risk and improved quality of life in patients with schizophrenia.

\section{Dietary folate intake, homocysteine levels and MTHFR mutation detection in South African patients with depression: Test development for clinical application D Delport, ${ }^{*} \mathrm{~N}$ van der Merwe, R Schoeman, M J Kotze \\ Department of Pathology, Faculty of Medicine and Health Sciences, University of Stellenbosch, Cape Town, South Africa \\ *darnielledelport@gmail.com}

Background. High homocysteine levels, associated with an increased risk of depression, are influenced by age, gender, genetic variation and environmental factors. Reduced folate status in the presence of the low-penetrance Methylenetetrahydrofolate reductase (MTHFR) C677T (rs1801133) mutation is an important cause of elevated homocysteine levels, reflecting dysfunction of the epigenetic process of DNA methylation. This pathway is crucial for the synthesis of noradrenaline and serotonin in the brain.

Objective. The role of MTHFR C677T was investigated in patients diagnosed with depression, compared with controls without a personal and family medical history of overlapping stress/anxiety and depressive disorders.

Methods. Folate scores were determined in 333 white individuals aged 18 - 60 years. As an overlapping study showed a significant increase in homocysteine levels with a reduction in the folate score, determination of homocysteine levels was extended to a subgroup of 96 patients with a definite diagnosis of depression. High-throughput real-time polymerase chain reaction (RT-PCR) analysis was performed to determine genotype distribution in 96 patients and 237 controls.

Results. A low folate score was identified in 96 patients and 237 controls. In patients diagnosed with depression, $42.7 \%$ had relatively high homocysteine levels $(>10 \mu \mathrm{mol} / \mathrm{l})$. Of the patients (17 male, 79 females) with a definite clinical diagnosis of depression 11.5\% were homozygous for the T allele of MTHFR C677T, 41.7\% heterozygous and $45.8 \%$ wild type. Similar genotype frequencies were observed in the control group, including 83 males and 153 females.

Conclusion. High homocysteine levels identified in 41 of the patients diagnosed with depression could largely be explained by a combination of genetic and environmental risk factors. MTHFR genotyping may facilitate the clinical management of patients with depression, through early detection of hyper-responders to known environmental influences. There is evidence of shared genetic vulnerability for many chronic diseases (including depression) mediated by the MTHFR 677 T allele.

\section{The use of exome sequencing for antipsychotic pharmacogenomic applications in South African schizophrenia patients}

B Drögemöller, ${ }^{1 *}$ D Niehaus, ${ }^{2}$ G Wright,,${ }^{1,3}$ B Chiliza, ${ }^{2}$ L Asmal, ${ }^{2}$

R Emsley, ${ }^{2}$ L Warnich ${ }^{1}$

${ }^{1}$ Department of Genetics, Stellenbosch University, Cape Town, South Africa

${ }^{2}$ Department of Psychiatry, Stellenbosch University, Cape Town, South Africa

${ }^{3}$ South African Bioinformatics Institute, University of the Western Cape, Cape Town, South Africa

*14337185@sun.ac.za

Background. Schizophrenia creates an immense socioeconomic burden, which is aggravated by poverty in low- and middle-income countries. Current treatments have substantial limitations. As African populations have been under-represented in genome-wide research to date, this study aims to identify unique patterns of genomic variation that may affect antipsychotic response in South African patients.

Methods. The study collected a cohort of 104 South African firstepisode schizophrenia patients, who have been uniformly treated and assessed for 2 years. A subset of age-, sex- and ethnicity-matched antipsychotic non-responders (4 unrelated individuals and 1 sibpair) and responders $(n=5)$ were identified for exome sequencing. The generated exome data was analysed utilising BWA, SAMtools, GATK, SeattleSeq Annotation and VAAST. Thereafter a panel of 384 variants, including ancestry informative markers, was prioritised for genotyping in a larger cohort of 480 schizophrenia patients.

Results. Using a variety of options in VAAST to model antipsychotic response, we identified a list of genes that may contribute to antipsychotic treatment response. Variation predicted to alter the protein products of these genes and other candidate genes was prioritised for genotyping in the entire cohort.

Conclusion. By utilising a well-characterised cohort and identifying variation affecting patients on extreme ends of the treatment response phenotypic spectrum, we identified a unique panel of variation that may contribute to antipsychotic response in schizophrenia patients. These results highlight the important role that rare variation may play in antipsychotic treatment response outcomes.
The effects of HIV on the ventral-striatal reward system $\underline{\mathrm{S} \text { du Plessis, }},{ }^{1 \star} \mathrm{M}$ Vink, ${ }^{2} \mathrm{~J}$ Joska, ${ }^{5}$ E Koutsilieri, ${ }^{3} \mathrm{C}$ Scheller, ${ }^{3}$ B Spottiswoode, ${ }^{6}$ D Stein, ${ }^{4,5}$ R Emsley ${ }^{1}$
${ }^{1}$ Department of Psychiatry, Stellenbosch University, Cape Town, South Africa
${ }^{2}$ Rudolf Magnus Institute of Neuroscience, University of Utrecht, The Netherlands
${ }^{3}$ Institute for Virology and Immunobiology, University of Würzburg, Würzburg, Germany
${ }^{4}$ Medical Research Council Unit on Anxiety and Stress Disorders, Stellenbosch University, Cape Town, South Africa
${ }^{5}$ Department of Psychiatry, University of Cape Town, South Africa 
${ }^{6}$ MRC/UCT Medical Imaging Research Unit, Department of Human Biology, University of Cape Town, South Africa

*stefandup@sun.ac.za

Background. HIV-associated neurocognitive disorders (HAND) present with behavioural, motor and cognitive symptoms, and are thought to affect the frontal-subcortical system, but the involvement of the various sub-networks of this system has not been fully elucidated.

Objective. To examine the ventral-striatal reward system in HIVpositive subjects, a system essential for everyday emotional processing and goal-directed behavior.

Methods. 17 drug- and smoking-naïve HIV-positive subjects (13 female; mean age 31 years; mean $\mathrm{CD}^{+} 66$ cells/ $\mu \mathrm{l} ; 2$ on highly active antiretroviral therapy (HAART)) along with 17 gender-, age- and education-matched controls, performed a monetary incentive delay functional magnetic resonance imaging (fMRI) task, known to activate the ventral-striatal reward network. Subjects with severe depression were excluded. Subjects were presented with reward, fixation, target and reward feedback cues, and required to respond as fast as possible to the target cue. Task difficulty was adjusted according to individual performance to assure an equal amount of reward and no-reward trials.

Results. Both groups received an equal monetary reward (control mean \pm standard deviation (SD) R139 \pm 3 ; HIV mean \pm SD R $137 \pm 4$, $p=0.729$ ). HIV-positive subjects showed significantly increased activation in their left ventral-striatum $(p=0.048)$ and bilateral superior orbito-frontal regions (left $p=0.033$; right $p=0.045$ ) on reward feedback. In general, HIV-positive subjects demonstrated larger relative activation upon receiving reward, and larger deactivations when they did not receive a reward.

Conclusion. Our findings provide evidence of potential viral effects on the reward system, with implications for how HIV-positive patients respond to environmental stimuli. Further work is needed to explore the impact of this on behavior, and whether it is a state or trait phenomenon.

\section{Xenomelia relates to asymmetrical insular activity: A case study in fMRI \\ $\underline{\text { S du Plessis }},{ }^{1 *}$ M Vink, $^{2}$ L Asmal $^{1}$ \\ ${ }^{1}$ Department of Psychiatry, Stellenbosch University, Cape Town, South Africa \\ ${ }^{2}$ Rudolf Magnus Institute of Neuroscience, University of Utrecht, The Netherlands \\ *stefandup@sun.ac.za}

Background. Xenomelia is a rare condition where an otherwise healthy individual experiences one or more of their limbs to be 'foreign', 'intrusive' or 'over present' and often expresses the desire to amputate the affected limb. The right inferior parietal lobule is implicated suggesting an abnormality in the integration of basic sensory information into a coherent 'body schema'. Evidence for higher cortical function, responsible for the generation of a more subjective or emotional body image, is still lacking. To our knowledge this condition has never been examined using functional magnetic resonance imaging (fMRI). A 48-year-old man was referred to the Joint Psychiatry Neurology Clinic at Tygerberg Hospital with constant thoughts since early childhood that his right leg below mid-thigh feels foreign. Emotional response but not physical symptoms are alleviated on antidepressants. There was no other axis 1 or 2 comorbidity.

Method. fMRI was performed on the 3T scanner. The subject underwent alternating, bilateral somatosensory soft touch stimulation to the dorsal hand and anterior lower limb. A block design was followed with alternating 30-second stimulation periods for two 10-minute sessions.

Results. The subject reported that symptoms of xenomelia increased dramatically due to stimulation during the scan. Contrasting right arm with left arm activation revealed a cluster in the contra-lateral somatosensory cortex (FWE $p>0.05$ ) as expected. The same contrast in the lower limbs also revealed comparable activation in the contralateral somatosensory cortex. This contrast also yielded a large area of activation in the left middle insular cortex, and smaller clusters in the parietal lobe and the right orbito-frontal cortex.

Conclusion. Subject showed asymmetrical activation as expected in the superior-parietal lobe. A novel finding was asymmetrical higher cortical activations in the limbic as well as the middle insular regions contralateral to the affected limb, regions thought to be involved in higher cortical body representation. We therefore propose that not only basic body representations are involved in this disorder, but also higher cortical representations. These results support the classification of the xenomelia as a neuropsychiatric body identity disorder.

Maternal mental health: A prospective naturalistic study of the outcome of pregnancy in women with major psychiatric disorders in an African country

E du Toit, ${ }^{1 *}$ L Koen, ${ }^{2,3}$ D Niehaus, ${ }^{2,3}$ B Vythilingum, ${ }^{4}$ E Jordaan, ${ }^{5}$

J Leppanen $^{6}$

${ }^{1}$ Sophia Maternal Mental Health Clinic, Panorama Psychiatric Clinic, Cape Town, South Africa

${ }^{2}$ Stikland Psychiatric Hospital, Cape Town, South Africa

${ }^{3}$ Department of Psychiatry, Faculty of Medicine \& Health Sciences,

University of Stellenbosch, Cape Town, South Africa

${ }^{4}$ Divison of CL Psychiatry, Department of Psychiatry, University of Cape Town, South Africa

${ }^{5}$ Biostatistics Unit, Medical Research Council, Cape Town, South Africa

${ }^{6}$ Tampere Center for Child Health Research, University of Tampere

School of Medicine, Tampere, Finland

*elsadutoit1@gmail.com

Background. Maternal mental health vulnerability exists in the pre- and postpartum periods, especially in mothers suffering from pre-existing mental illness. South Africa has no uniform management plan for this, with ad hoc treatment plans and lack of a central advisory service impeding optimal management of this vulnerable group. State and private clinics in Cape Town established a collaborative network to formulate a holistic, uniform approach within available resources.

Methods. This preliminary report deals with the first 38 pregnant female mental health users referred to the maternal mental health clinic at Stikland Hospital. Data were collected as part of patient reviews during care-as-usual visits, and included reports and findings as per treatment-as-usual obstetrics and gynaecology review. 
Results. The 38 participants (mean age 29 years) presented on average at 17 weeks pregnancy duration. Nine were primigravida (mean gravida $n=3$ ) and only $21 \%$ were planned pregnancies. Two participants presented after a suicide attempt with $50 \%$ of the cases reporting a previous history of suicide attempts. The most common psychiatric diagnoses were bipolar mood disorder (26\%), major depressive disorder (18\%), substance misuse disorders (21\%) and schizophrenia (11\%). Pregnancy outcomes include 1 miscarriage, 1 stillbirth and 12 live births.

Conclusion. Preliminary results indicate that mental health users present relatively late to psychiatric services, that unplanned pregnancy is common in this population and a concerning prevalence of substance-use disorders. The optimal management of maternal mental health during pregnancy and in the immediate postpartum period is therefore vital.

\section{Prefrontal cortical thinning and subcortical volume decrease in HIV-positive children with encephalopathy}

JP Fouche, ${ }^{1,2,3 *}$ B Spottiswoode,,${ }^{1,4}$ K Donald, ${ }^{5}$ D Stein, ${ }^{2}$ J Hoare ${ }^{2}$

${ }^{1}$ Department of Human Biology, University of Cape Town, Cape Town, South Africa

${ }^{2}$ Department of Psychiatry and Mental Health, University of Cape Town, Cape Town, South Africa

${ }^{3}$ Department of Psychiatry, University of Stellenbosch, Cape Town, South Africa

${ }^{4}$ Department of Radiology, University of Stellenbosch, Cape Town, South Africa

${ }^{5}$ Division of Developmental Paediatrics, University of Cape Town, South Africa

*jfouche@sun.ac.za

Background. Mother-to-child transmission of HIV represents a severe problem, especially in sub-Saharan Africa. About $50 \%$ of HIVinfected children present with central nervous system symptoms in the initial phase of infection. Previous structural imaging research in HIV-positive children has identified cortical atrophy, basal ganglia calcifications and white matter lesions. A number of such children also present with encephalopathy. However, there are no morphometric data on changes in cortical thickness for HIV-positive children.

Objective. To investigate changes in cortical thickness and subcortical volume between HIV-positive children with and without encephalopathy and healthy HIV-negative controls.

Methods. The study group comprised 45 HIV-positive patients (31 without encephalopathy, 14 with encephalopathy) on highly active antiretroviral therapy (HAART) and 25 controls, aged 6 - 14 years. There was no evidence of any other infection and patients were not exposed to any substances in utero. A multi-echo magnetizationprepared rapid gradient echo (MP-RAGE) T1-weighted image was acquired on a 3T magnetic resonance imaginf (MRI) scanner. Cortical and sub-cortical regions were delineated using Freesurfer version 5.1. Cortical thickness values were compared using SPSS version 20.0.

Results. Age, gender and level of education were controlled for. For the cortical regions, significant thinning $(p<0.05)$ was found in the left inferior precentral, medial parahippocampal, superior frontal gyri and right inferior frontal gyrus of HIV-positive patients with encephalopathy, compared with controls. Subcortical volumes were significantly decreased $(p<0.05)$ in the left amygdala and right accumbens of HIV-positive subjects without encephalopathy and in the bilateral hippocampus and left amygdala of HIV-positive subjects with encephalopathy, versus controls. There was a negative correlation of $\mathrm{CD} 4^{+}$count with the hippocampus in the HIV-positive group.

Conclusion. Thinning in frontal grey matter regions and decreased subcortical volume are consistent with previous neurological findings, which demonstrated general frontal atrophy in HIV-positive children. These regions are part of the frontal-striatal circuit that is affected in HIV-positive adults.

\section{${ }^{1} \mathrm{H}$-magnetic resonance spectroscopy metabolites in schizophrenia}

F Howells, ${ }^{1 *}$ J Hsieh, H Temmingh, D J Stein ${ }^{2}$

${ }^{1}$ Groote Schuur Hospital, Cape Town, South Africa

${ }^{2}$ Department of Psychiatry and Mental Health,

University of Cape Town, South Africa

^howellsfleur@gmail.com

Background. The neurobiological mechanisms that result in the development and persistence of schizophrenia remain elusive.

Methods. ${ }^{1} \mathrm{H}$-Magnetic resonance spectroscopy permits insight into metabolic dysfunction present in mental disease, including schizophrenia. This study included magnetic resonance imaging (MRI) scanning of 15 individuals with the diagnosis of schizophrenia and 15 demographic matched-controls. We assessed the metabolic profiles of 3 brain areas known to be involved in schizophrenia: anterior cingulate cortex, dorsolateral prefrontal cortex, and thalamus. Results. Our results show disruption of metabolism in the anterior cingulate cortex and thalamus. Within the anterior cingulate cortex, we found decreased $\mathrm{N}$-acetyl-aspartate metabolites and decreased creatine metabolites. Within the thalamus we found increased creatine metabolites, increased myo-inositol, and increased glutamate and glutamine.

Conclusions. These results show differential metabolism in cortical v. sub-cortical brain structures in schizophrenia. Within the anterior cingulate cortex there is decreased neuronal integrity and viability and decreased energy, while in the thalamus there is increased energy and increased neuronal excitability. These results suggest a mismatch in cortical and thalamic metabolism.

\section{Hypothesis for the development of persistent methamphetamine-induced psychosis \\ L Hsieh, ${ }^{*}$ D J Stein, F M Howells \\ Department of Psychiatry and Mental Health, University of Cape \\ Town, South Africa \\ *humbleambition@gmail.com}

Background. Chronic methamphetamine (MA) abuse commonly leads to psychosis, with symptoms indistinguishable from those seen in paranoid schizophrenia. These symptoms can persist without 
continued use of MA. Clinical similarities between MA-induced psychosis (MAP) and schizophrenia suggest that MAP may be a useful model for schizophrenia. There is, however, no biological hypothesis of the development of MAP, and its persistence.

Method. A literature review was conducted of research involving methamphetamine effects in humans and animals. Keywords included: 'methamphetamine neurotoxicity', 'methamphetamine psychosis,' 'methamphetamine cortex' and 'schizophrenia mechanism'. The results led to a deeper search for biological mechanisms, using keywords including 'cortical glutamate and gamma-aminobutyric acid (GABA),' 'cortical GABA neurons', 'GABA neuron toxicity' and 'GABA vulnerability'.

Results. The literature search found a clear line of biological evidence that links methamphetamine use and abuse to cortical gammaaminobutyric acid (GABA) dysfunction. The present hypothesis asserts that methamphetamine use, through polysynaptic pathways, causes an overflow of glutamate to the cortex, and stimulates neurotoxically vulnerable cortical GABAergic interneurons. Neurotoxicity of the interneurons causes loss of neurophils and permanent GABA dysfunction, which in turn exacerbates glutamate and dopamine dysregulation. Each of these neurobiological dysfunctions have been researched independently and have shown contributions toward the development of psychosis.

Conclusion. This is a novel hypothesis for the development of MAP and its persistence. Additional direct evidence is needed to demonstrate decreased basal GABA concentrations, decreased GABA receptor expression, or decreased neuropil in the cortex after MA use, especially persistent MA use.

Culture, religion, spirituality and psychiatric practice: The SASOP Spirituality and Psychiatry Special Interest Group

\section{Action Plan for 2012 - 2014}

\section{B Janse van Rensburg}

Department of Psychiatry, University of the Witwatersrand,

Johannesburg, South Africa

bernardj@gpg.gov.za

The proposed vision, mission and practice values for the South African Society of Psychiatrists (SASOP) Spirituality and Psychiatry Special Interest Group for 2012 - 2014 are:

Vision: An appropriate integrated bio-psycho-social-spiritual approach to the assessment and management of clinical psychiatric problems

Mission: To incorporate the role of defined spirituality into the existing bio-psycho-social approach to local practice and teaching of specialist psychiatry - within specific professional and ethical boundaries - while accommodating all cultural-religious traditions and belief systems in the heterogeneous South African society equally.

Values: (i) Education and communication: providing health education initiatives aimed at psychiatrists, patients, patient carers, students and cultural/religious practitioners; (ii) Protection: identifying and preventing abuse, neglect, or delay of appropriate treatment of individuals with psychiatric disorders within traditional health practice or other cultural and religious systems.
The proposed objectives for this period are:

1. Equal accommodation: To ensure that if the principle of incorporation of the role of spirituality is considered, that all cultural-religious traditions and belief systems, including nonreligious/atheistic perspectives, are equally accommodated

2. Application of definitions: To ensure that appropriate definitions of culture, religion and spirituality are considered and applied in different service, practice and training environments

3. Guidelines: To develop and implement appropriate guidelines on the role of spirituality for:

- integrated clinical care and service delivery requirements

- integrated training of local undergraduate and postgraduate students in psychiatry

- the ethical integration of spirituality into the local practice of psychiatry within the professional scope of the discipline

- the appropriate integrated referral of patients and collaboration between psychiatrists/psychiatric services and spiritual professionals.

\section{Cocaine reduces the efficiency of dopamine uptake in a rodent model of attention-deficit/hyperactivity disorder: An in vivo electrochemical study}

L Kellaway, ${ }^{1 *}$ J S Womersley, ${ }^{1}$ D J Stein, ${ }^{2}$ G A Gerhardt, ${ }^{3}$ V A Russell ${ }^{1}$

${ }^{1}$ Department of Human Biology, University of Cape Town, South Africa

${ }^{2}$ Department of Psychiatry, University of Cape Town, South Africa

${ }^{3}$ Department of Anatomy and Neurobiology, Center for Microelectrode Technology, Morris K. Udall Parkinson's Disease Research Center of Excellence, University of Kentucky Chandler Medical Center, USA *lauriston.kellaway@uct.ac.za

Background. Attention-deficit/hyperactivity disorder (ADHD) is a developmental disorder characterised by age-inappropriate levels of inattention, impulsivity and hyperactivity. The spontaneously hypertensive rat (SHR) is a well-characterised animal model of ADHD that displays dopaminergic dysregulation, which is hypothesised to be a cause of ADHD. Developmental stress is associated with various psychiatric conditions later in life and can be modelled by maternal separation in animals, where it produces subsequent dopaminergic dysregulation.

Methods. Maternally separated (MS) SHR and control Wistar-Kyoto (WKY) litters were removed from the dam for 3 hours per day from postnatal days 2 to 14 , and compared with non-maternally separated (NMS) litters. In vivo chronoamperometry was used for real-time measurement of dynamic changes in pressure-ejected dopamine in the striatum, providing a measure of dopamine transporter (DAT) function. Dopamine was ejected every 5 minutes to attain reproducible peaks of amplitude $0.75 \mu \mathrm{M}-1.5 \mu \mathrm{M}$. We calculated the averages of 3 peaks before and after the peripheral injection of cocaine, a DAT inhibitor. Difference scores were calculated by subtracting the post-cocaine administration values from the pre-cocaine administration values.

Results. A repeated measures analysis revealed that peak amplitude and area were significantly increased following cocaine administration across all experimental groups. Maternal separation significantly increased the time required for the amplitude to fall to $50 \%$ of peak value. This difference was due to an effect of MS in SHR, which was not observed in WKY. 
Conclusions. Dopamine peak amplitude and area were significantly increased after cocaine administration in both SHR and WKY. Maternal separation impaired DAT function in SHR, increasing the time required to clear dopamine from the extracellular fluid. This is consistent with previous findings that MS decreased the efficiency of dopamine uptake by DAT in SHR striatum.

\section{Kleine-Levin syndrome: Case in an adolescent psychiatric unit}

\section{A Lachman}

Department of Psychiatry, Tygerberg Hospital, Stellenbosch University, Cape Town, South Africa

anusha@sun.ac.za

Background. Kleine-Levin Syndrome (KLS) is an exceptionally rare, recurrent condition characterised by a triad of hypersomnia, hyperphagia, and behavioural disturbances (hypersexuality, depression, irritability, derealisation). It occurs typically in adolescent males who are often misdiagnosed with depression, bipolar disorder, psychosis or seizure disorders and who return to normal between attacks. The disorder is at the interface of neurology and psychiatry, and no objective biochemical, neuropathological or structural imaging alterations have been identified.

Case. This is the first case of typical KLS documented in South Africa and describes a 15-year-old male with no previous psychiatric or medical history, no substance abuse and no significant family history, who presented over a 2-year period with recurrent episodes of hypersomnia, hypersexuality and hyperphagia. Following normal results of extensive investigations, he was initially diagnosed with a postictal psychotic disorder and oligoarthritis. Symptoms resolved with an anticonvulsant, antipsychotic (risperidone) and salazopyrine. Three months later he presented with hypersomnia, blurred vision and irritability. Investigations again were normal. He settled with low dose of risperidone and returned to his previous level of functioning, until a repeat episode of hypersomnia warranted readmission. A diagnosis of bipolar disorder and psychosis was excluded on the basis of Diagnostic and Statistical Manual (DSM) IV TR3 criteria, and there were no residual symptomatology inter-episodically. After extensive neurological consultations, a diagnosis of KLS was made.

Conclusions. KLS may be an elusive diagnosis because of its infrequent presentation, and symptoms may be confused with other disorders. Our patient was diagnosed with seizures despite normal investigations and unnecessarily prescribed anticonvulsants. This complicated his clinical status, requiring further investigations. In any adolescent (especially males) presenting with recurrent periodic hypersomnia associated with psychiatric/behavioural issues with normal inter-episode functioning, after excluding medical or psychiatric history, KLS should be considered on the differential.

Increased inflammatory stress influences specific clinical, lifestyle and therapeutic variables in patients receiving treatment for stress, anxiety or depressive symptoms $\underline{\text { H Lückhoff, }}{ }^{\star}$ M Kotze, S Janse van Rensburg, D Geiger
Stellenbosch University, Cape Town, South Africa

•hilmarklausl@gmail.com

Background. Increased inflammation is indicated in the aetiology and development of various psychiatric illnesses, including chronic stress, anxiety and depression. By increasing the antioxidant enzyme expression, short-term antidepressant therapy appears to modulate this, while anti-inflammatory agents may augment treatment in resistant cases. Classifying patients based on c disease characteristics aids in predicting increased inflammatory stress. Polymorphisms in various genes regulating the inflammatory response have been implicated in depression, . Nascent literature suggests an association with depression risk, as well as a decreased therapeutic response to antidepressants, while chronically distressed patients carrying the risk allele demonstrate increased plasma creatinine-reactive protein (CRP) levels. Correlations exist with the severity of sleep disturbances and morning fatigue, as well as an increased vulnerability towards labile anger in patients receiving exogenous interferon therapy.

Objective. To examine the influence of this dimorphism and highsensitivity CRP (hs-CRP) levels on specific clinical, lifestyle and therapeutic variables in patients receiving treatment for stress, anxiety or depressive symptoms.

Methods. Using questionnaires, we identified 90 patients receiving treatment for such symptoms. Biochemically, hs-CRP levels were measured as a marker of increased inflammation, while TNF- $\alpha$ genotype was determined using reverse transcription polymerase chain reaction (RT-PCR) methods.

Results. The presence of the TNF- $\alpha$ risk allele significantly influenced the age of onset of symptoms $(p<0.01)$. Serum hs-CRP levels were significantly higher in those subjects who reported treatment-related side-effects $(p<0.01)$. These subjects were also significantly more likely to be smokers.

Conclusions. Increased inflammatory stress significantly affects specific clinical, lifestyle and therapeutic variables in stress, anxiety and depressive disorders. Classifying patients according to certain illness characteristics may predict such changes.

\section{Catatonia: An eight-case series report \\ M Mabenge, ${ }^{1 \star} \mathrm{Z}$ Zingela, ${ }^{1} \mathrm{~S}$ van $\mathrm{Wyk}^{2}$}

${ }^{1}$ Department of Psychiatry, Port Elizabeth Hospital Complex, Port Elizabeth, South Africa

${ }^{2}$ Department of Psychiatry, Uitenhage Provincial Hospital, Uitenhage, South Africa

*mze@mweb.co.za

Background. Catatonia is an important but often neglected syndrome and many conditions can present with catatonia. Lesser-known signs are often missed, but rapid and accurate detection is crucial for effective management. Early intervention can prevent progression to morbid sequelae, including malignant catatonia, a life-threatening but treatable neuropsychiatric disorder, which can have a positive outcome if detected early. Approximately $50 \%$ of catatonia patients have mood disorders, whereas 10 - 20\% have schizophrenia. The remaining cases are associated with substances, medical, neurological or other psychiatric disorders. 
Objective. This case series highlights the presentations, diagnoses, comorbidities, management and outcomes of 8 patients admitted with catatonia to 2 acute psychiatry wards in the Nelson Mandela Bay Metro.

Method. A retrospective analysis of case notes included clinical presentation, duration of symptoms, symptom severity, clinical diagnosis, special investigations, management and outcome. Common factors influencing positive and negative outcomes were identified.

Results. Out of 8 patients ( 4 females), 4 had a mood disorder, 2 had psychosis due to HIV, 1 had schizophreniform disorder and 1 had neurosyphylis. Most had raised serum creatinine kinase levels and correspondingly low serum iron. Two female patients also had microcytic anaemia. Multimodal management included psychiatry, internal medicine, physiotherapy and dietetics. Acute psychiatric intervention included intravenous and oral benzodiazepines and electroconvulsive therapy. Six patients were transferred to the local psychiatric hospital once medically stable. One female was managed in a medical ward for 3 months before discharge home. Two patients developed chronic contractures and 1 had a fatal outcome.

Conclusion. This report adds to the body of knowledge regarding presentation and management of catatonia. A number of factors were associated with severity, response to treatment and outcome. It is concerning that morbidity and mortality was relatively high.

\author{
Relationship between anxiety sensitivity and childhood \\ trauma in a random sample of adolescents from secondary \\ schools in Cape Town \\ L Martin, ${ }^{1 *}$ M Viljoen, $^{2}$ S Seedat ${ }^{1}$ \\ ${ }^{1}$ Department of Psychiatry, Stellenbosch University, Cape Town, South \\ Africa \\ ${ }^{2}$ Department of Physiological Sciences, Stellenbosch University, Cape \\ Town, South Africa \\ *lmartin@sun.ac.za
}

Background. A variety of aetiological factors contribute to the development and persistence of anxiety disorders. Individual trait characteristics, such as anxiety sensitivity (AS) and trait anxiety have been found to be elevated in those with anxiety disorders. Childhood trauma (CT) is associated with an increased risk for these disorders. The combination of AS and CT may increase the risk for early-onset anxiety disorders and subsequent comorbidity.

Objective. To: (i) assess levels of AS in the sample overall and by gender; (ii) report on the risk/psychopathology profile as well as protective factors; (iii) assess the relationship between AS and CT; and (iv) determine whether CT uniquely predicts AS scores and whether resilience and coping modify this prediction.

Methods. Learners from a representative sample of Cape Town secondary schools ( $n=746$, mean age 16 years), completed a battery of self-report instruments that assessed risk factors (AS, trait anxiety, CT, depression, and alcohol and drug use), and protective factors (resilience and coping orientation). Most participants were black and almost $60 \%$ were female.

Results. We found no difference between males and females in terms of levels of CT, resilience and coping behaviour; however, females had significantly higher levels of AS, trait anxiety and depression. Males had significantly higher levels of alcohol and drug use. CT did not uniquely predict AS - rather, depression and trait anxiety did.

Conclusion. Higher levels of CT in the presence of AS suggest a greater risk for both behavioural and psychological difficulties. Females are at greater risk than males for early-onset anxiety disorders despite the same rates of $\mathrm{CT}$, coping behaviour and resilience. The identification of specific risk markers for anxiety offer opportunities for early detection and intervention.

\section{'Making ethics real'. An overview of an ethics course presented by Fraser Health Ethics Services, BC, Canada Ш McCallaghan}

Psychiatrist in Private Practice

jjcobus14@gmail.com

This presentation will communicate the format and content of a 4-month ethics course presented by the Fraser Health Ethics Services, $\mathrm{BC}$, Canada. It will be in the form of a descriptive presentation regarding the format and content of the course. The course consisted of 8 modules and 5 workshops. Each module had a theme that was covered by a reading list, watching a DVD on the topic and a teleconference discussing the topic. The workshops were conducted under the supervision of the staff of the Fraser Health Ethics services. The themes for the modules were: duties or consequences; principlebased ethics; respect for autonomy; virtue ethics; diversity; moral distress; end of life; and resource allocation.The workshop topics were: introduction to bioethics/framework for ethical decision making; ethics consultation; skills practice; and system-level ethics consultation. The material presented should stimulate discussion on ethics and philosophy in mental health relevant to South Africa.

\section{Clozapine discontinuation rates in a public healthcare setting} M Moolman, ${ }^{1 *}$ W Esterhuysen, ${ }^{2}$ R Joubert, ${ }^{1}$ J C Lamprecht, ${ }^{1}$

\section{S Lubbe ${ }^{1}$}

${ }^{1}$ Medicine Usage in South Africa (MUSA), School of Pharmacy, NorthWest University, Potchefstroom Campus, South Africa

${ }^{2}$ Elizabeth Donkin Hospital, Forest Hill, Port Elizabeth, South Africa

*marisanmoolman@yahoo.com

Background. Early treatment discontinuation in patients with schizophrenia or schizophrenia-like disorders is very common, with about $50 \%$ of patients being non-adherent to treatment. According to the 18-month Clinical Antipsychotic Trial for Intervention Effectiveness (CATIE) study, $74 \%$ of patients discontinued their antipsychotic medication, despite monthly monitoring. The results indicated that clozapine was more effective than the other second-generation antipsychotics: on average, patients stayed on clozapine for 10 months, and other antipsychotics for only 3 months. Discontinuing antipsychotic treatment is associated with relapse, increased hospitalisation, symptom exacerbation, poor long-term course of illness, and higher treatment 
costs. Reasons for poor adherence are complex, but could be related to a hidden burden of adverse effects and poor illness insight.

Objective. To determine the observed discontinuation rates for clozapine, both 3 months and 6 months after discharge from hospital. Methods. Data were collected from the files of 65 patients discharged on clozapine between 1 December 2010 and 29 February 2012 from a public sector psychiatric hospital. Follow-up investigations were performed at community psychiatric clinics at both 3- and 6-month intervals.

Results. About 34\% ( $n=65)$ of patients had $>3$ previous admissions to a psychiatric hospital. Clozapine was previously prescribed for $52 \%(n=65)$. It was found that $71 \%(n=65)$ of patients were still on clozapine 3 months after discharge and 65\% $(n=65)$ were still on clozapine 6 months after discharge, discontinuation rates of $29 \%$ and $35 \%$ respectively.

Conclusion. The observed discontinuation rate exceeded that of the CATIE study. This is remarkable, since CATIE was conducted in a developed country whereas this study was conducted in a developing country with limited healthcare resources. The reason(s) for clozapine discontinuation need to be investigated, since clozapine's main indication is treatment-resistant schizophrenia.

\section{Retrospective review of clozapine monitoring in a public sector psychiatric hospital and associated clinics \\ M Moolman, ${ }^{1 \star}$ W Esterhuysen, ${ }^{2}$ R Joubert, ${ }^{1}$ JC Lamprecht, ${ }^{1}$ MS Lubbe ${ }^{1}$ \\ ${ }^{1}$ Medicine Usage in South Africa (MUSA), School of Pharmacy, North-West University, Potchefstroom Campus, South Africa \\ ${ }^{2}$ Elizabeth Donkin Hospital, Forest Hill, Port Elizabeth, South Africa *marisanmoolman@yahoo.com}

Background. Clozapine has shown superior efficacy over conventional antipsychotics (for both the positive and negative symptoms of schizophrenia) as well as proven efficacy in the treatment of refractory schizophrenia. International guidelines recommend both the haematological and metabolic monitoring of clozapine. The Standard Treatment Guidelines, Hospital Level for Adults, suggest that a psychiatrist should initiate clozapine therapy and that haematological monitoring should be performed according to the package insert. However, there are no South African guidelines recommending the metabolic monitoring of clozapine.

Objective. To assess the safe and effective use of clozapine, by investigating the monitoring patterns followed in a tertiary hospital, using a public sector psychiatric hospital and community psychiatric clinics as a model.

Methods. A quantitative, retrospective drug use review of clozapine was performed in a public sector psychiatric hospital. Data were collected from the files of 65 patients discharged on clozapine between 1 December 2010 and 29 February 2012. Haematological and metabolic monitoring of clozapine was compared to recommended national and international guidelines. Follow-up investigations were performed at the clinics, both 3 and 6 months after discharge from hospital.

Results. Non-compliance with the recommended guidelines for follow-up haematological monitoring occurred in $77.4 \%(n=62)$ of patients in the hospital setting and $95.7 \%(n=47)$ during the 3-month follow-up. Non-compliance with the recommended guidelines for follow-up metabolic monitoring occurred in all the observed patients $(n=62)$ in the hospital setting and $46.9 \%(n=49)$ during the 3-month follow-up.

Conclusion. Clozapine was inadequately monitored. A guidance document should be developed and included in patients' files to assist in the safe and effective monitoring of clozapine in South Africa's public health sector.

Association of an iron-related TMPRSS6 genetic variant c.2207 C>T (rs855791) with functional iron deficiency and its effect on multiple sclerosis risk in the South African population

K Moremi,${ }^{1,2 *}$ S J van Rensburg, ${ }^{1,2}$ L R Fisher, ${ }^{1,3}$ W Davis, ${ }^{1,2}$

F J Cronje, ${ }^{4}$ M Jalali Sefid Dashti, ${ }^{5}$ J Gamieldien, ${ }^{5}$ D Geiger, ${ }^{1,3}$ M Rensburg, ${ }^{1,2} \mathrm{R}$ van Toorn, ${ }^{6} \mathrm{M}$ J de Klerk, ${ }^{1,2} \mathrm{G}$ M Hon, ${ }^{7}$ T Matsha, ${ }^{7}$ S Hassan, ${ }^{7}$ R T Erasmus, ${ }^{1,2}$ M Kidd, ${ }^{8}$ M J Kotze ${ }^{1,3}$

${ }^{1}$ National Health Laboratory Service, Cape Town, South Africa

${ }^{2}$ Department of Chemical Pathology, Stellenbosch University, Cape Town, South Africa

${ }^{3}$ Department of Anatomical Pathology, NHLS and Stellenbosch University, Cape Town, South Africa

${ }^{4}$ Interdisciplinary Health Sciences, Stellenbosch University, Cape Town, South Africa

${ }^{5}$ Knowledge Integration \& Biomarker Discovery, South African National Bioinformatics Institute, University of the Western Cape, South Africa

${ }^{6}$ Paediatric Medicine and Child Health, Stellenbosch University, Cape Town, South Africa

${ }^{7}$ Department of Bio-Medical Sciences, Faculty of Health and Wellness Science, Cape Peninsula University of Technology, Cape Town, South Africa

${ }^{8}$ Centre for Statistical Consultation, Department of Statistics and Actuarial Sciences, Stellenbosch University, South Africa

*kelebogilemor@gmail.com

Background. Lower iron concentrations are strongly suspected to worsen the symptoms of multiple sclerosis (MS), since iron availability is a prerequisite for myelin synthesis. Structural variations in matriptase-2, one of the major regulatory proteins of the iron absorption pathway, result in functional iron deficiency and are encoded by the TMPRSS6 gene.

Objective. To investigate the distribution of a common variant in the TMPRSS6 gene, c.2207C>T(rs855791), in MS patients in South Africa's Western Cape province.

Methods. The study population consisted of 107 patients diagnosed with MS according to the McDonald criteria and 85 controls without neurological disease. DNA was extracted from all samples for genotyping of functional single nucleotide polymorphisms (SNPs) in the TMPRSS6 gene, c.2207C > T (rs855791). High-throughput real-time polymerase chain reaction (RT-PCR) methodology was applied using the Applied Biosystems (ABI) TaqMan assay, ABI 7900HT, following analytical validation against direct sequencing as the gold standard.

Results. There were no significance differences in TMPRSS6 c.2207C > T variant frequency distributions between MS patients and controls $(p=0.893)$. However, there was a significant difference between 
homozygosity for the iron-deficiency risk $\mathrm{T}$ allele and wild type $\mathrm{C}$ allele in the study population. Serum ferritin levels were significantly lower in patients than controls $(p=0.001)$, with a significant difference between homozygous $\mathrm{T}$ allele and wild-type $\mathrm{C}$ allele $(\mathrm{p}=0.033)$. The $\mathrm{T}$ allele was consistent with lower levels of serum iron $(p=0.014)$ and lower $\%$ transferrin saturation $(p=0.039)$. In the MS group the T-allele was associated with an earlier age of diagnosis and presentation of first symptoms, although this was not statistically significant.

Conclusion. These findings suggest that the c.2207C>T variant could contribute to increased risk for developing iron deficiency in MS. The results confirm the link between the TMPRSS6 genetic variant c. 2207C $>\mathrm{T}$ and reduced iron levels in MS. Together with measurement of blood iron parameters, this could help identify a subgroup of MS patients with a lack of iron bioavailability, which may account for worsening of disease symptoms.

\section{Identifying molecular mechanisms of apormophine- induced addictive behaviours}

\section{Z Ndlazi, ${ }^{*}$ W Daniels, M Mabandla}

Department of Human Physiology, School of Medical Sciences, Faculty of Health Sciences, University of Kwa-Zulu Natal, Durban, South Africa *ndlaziz@ukzn.ac.za

Background. Some 15\% of South Africa's population have drug issues and drug abuse is costing the country close to R20 billion annually, threatening the country's future. Addiction is a chronic relapsing disorder characterised by constant drug-seeking and -taking behaviours. It is a repetitive self-administration of addictive substances despite the knowledge of the severe consequences. The addiction process involves 2 important dopamine pathways, the mesolimbic and mesocortical pathways

Objective. We hypothesise that chronic exposure to drugs of abuse leads to prolonged elevation in dopamine and glutamine levels that are toxic to the brain. Glutamate transmission is central in learning and memory and we postulate that these processes are similar to the neuroplastic changes necessary for the development of addictive behaviour. Administration of a glutamatergic antagonist will thus prevent development of such behaviour.

Methods. A total of 48 adult male Sprague-Dawley rats were used to characterise the addictive behaviour model and understand the role of glutamate receptors in the development of this behaviour. Animals were subjected to saline, apormophine subcutaneous injections and memantine intraperitoneal injections at varying doses. A condition place preference paradigm was subsequently used to determine the rewarding effects of apormophine and the effectiveness of memantine as a glutamate antagonist. Animals were sacrificed and brain tissue was collected.

Results. Memantine did not change the time spent on the drug-paired compartment, thus suggesting that both $5 \mathrm{mg} / \mathrm{kg}$ and $10 \mathrm{mg} / \mathrm{kg}$ doses of memantine did not block the rewarding effects of apomorphine.

Conclusion. The various doses of memantine were not adequate enough to supress the apormorphine-induced conditioned place preference; hence the study couldn't confirm the role of glutametergic system in the acquisition of apormophine reward.
Effects of lifestyle factors and biochemistry on the major neck blood vessels in patients with multiple sclerosis $\underline{\text { M Nelson, }}{ }^{1 *}$ S J van Rensburg,,${ }^{2,3}$ M J Kotze, ${ }^{2,4}$ F Isaacs, ${ }^{1}$ S Hassan ${ }^{1}$ ${ }^{1}$ Department of Nursing and Radiography, Faculty of Health and Wellness Sciences, Cape Peninsula University of Technology, Cape Town, South Africa

${ }^{2}$ National Health Laboratory Service, Cape Town, South Africa ${ }^{3}$ Department of Chemical Pathology Stellenbosch University, Cape Town, South Africa

${ }^{4}$ Department of Anatomical Pathology, and Stellenbosch University, Cape Town, South Africa

*merlisacnelson@gmail.com

Background. Lifestyle factors such as smoking and exercise have a major impact on the circulatory system. Smoking is associated with atherosclerotic disease and risk of venous deep vein thrombosis (DVT) and is an independent risk factor for the diagnosis and progression of multiple sclerosis (MS).

Objective. To determine, with ultrasound, the prevalence of internal jugular vein (IJV) pathology and carotid artery disease in MS patients. Methods. Duplex ultrasound was used to demonstrate abnormal outflow patterns as well as structural abnormality of the IJVs in 29 MS patients. Carotid arteries were also assessed for atherosclerotic disease. Lifestyle and biochemistry assessments were available for 20 patients.

Results. A larger proximal and mid cross-sectional diameter (CSD) of the right IJV compared with the left ( $p=0.026$ and $p=0.023$, respectively) was demonstrated, which is similar to data found in the literature. Increased intima media thickness (IMT) was present in $13.33 \%$ of the non-smoking MS group and 20\% in the smoking MS group. IJV reflux was evident in $13.33 \%$ of the MS group. In the left carotid artery, IMT was significantly inversely associated with physical activity $(p=0.046)$. The smokers displayed significantly smaller cross-sectional diameters of the proximal and mid-left IJV compared to the non-smokers $(p=0.03$ and $p=0.02$ respectively). Fibrinogen (a risk factor for cardiovascular disease) was non-significantly associated with peak systoclic velocity and end diastolic velocity in the right carotid arteries $(p=0.05)$, suggesting reduced diameters of these arteries. No DVTs identified.

Conclusion. Early carotid artery disease was noted in smoking and non-smoking MS patients. However, the findings were nonsignificant. A significant reduction of cross-sectional diameters of the IJVs was evident in smoking MS patients, suggesting that smoking is not only a risk factor for atherosclerotic disease but could also be related to narrowing of the major neck veins.

\section{Nicotine protects against dopamine neurodegeneration and improves motor deficits in a Parkinsonian rat model} $\underline{\text { N Ngema, }},{ }^{*}$ P Ngema, M Mabandla, W Daniels

School of Laboratory Medicine and Medical Sciences, University of KwaZulu Natal, Durban, South Africa

*nomfundongema@yahoo.com

Background. Parkinson's disease (PD) is a progressive neurodegenerative disease of dopamine (DA) neurons. Oxidative stress has been shown to be 
the major component in the pathogenesis of PD. Nicotine is a stimulant mainly found in cigarettes and has been shown to exert protective action against neurotoxins. Nicotine exposure attenuates 6-OHDA-induced toxicity. An in vivo administration of nicotine to rodents with PD results in neuroprotective action. To further understand $\mathrm{PD}$, researchers have developed standardised animal models of PD.

Objective. The study aimed to investigate whether stress increases the vulnerability of dopamine neurons in adulthood by increasing the size of the lesion and if nicotine can attenuate the effects caused by the neurotoxin 6-OHDA.

Methods. In our study, male Sprague-Dawley(SD) rats were unilaterally lesioned by injection of the neurotoxin, 6-hydroxydopamine (6-OHDA) $(5 \mu \mathrm{g} / 4 \mu \mathrm{l})$, into the medial forebrain bundle (MFB) of the left hemisphere. The rats were divided into 4 groups: group A (6-OHDA only), group B (nicotine), group C (stress (7 days)) and group D (nicotine + stress (7 days)). We used restraint stress as a model to study behavioural changes in adult rats.

Results. Our results showed that nicotine has neuroprotective effects in both non-stressed and stressed rats. Stress attenuates the toxic effects of 6-OHDA and reduces the beneficial effects of nicotine. Nicotine reduced dopamine neuron degeneration in the non-lesioned hemisphere.

Conclusion. Nicotine is neuroprotective against dopamine neurodegeneration and exposure to stress enhances neuronal degeneration.

\section{Cognition: Probing anatomical substrates ... H Nowbath}

Psychiatrist in Private Practice

hemant@saol.com

'I think, therefore I am.'

- Rene Descartes, $17^{\text {th }}$ century French philosopher

'I believe that brain science will be to the $21^{\text {st }}$ century what quantum physics and DNA molecular biology were to the $20^{\text {th }}$ century.'

- William Newsome, one of a duo of neurobiologists leading a $\$ 100$ million project to crack the code of how the human brain is wired.

Despite rapid advances in the neurosciences in recent years some, core questions remain regarding cognition and cognitive function: How do we think? What brain mechanisms are involved in thought processes? What factors influence thought? How does the brain organise and retrieve information? This presentation explores the anatomical substrates of cognition. It reviews the relevant literature and examines historical and philosophical concepts relating to thought. The use of chess as a model for the study of cognitive skill is examined. Chess has been used to study memory, information processing and artificial intelligence. Neuroimaging studies allow assessment of brain function and facilitate an understanding of how information is processed. Various techniques - single-photon emission computed tomography (SPECT), positron emission tomography (PET), electroencephelography (EEG), functional magenetic resonance imaging (fMRI) - have made an invaluable contribution to neurosciences. Specific functions have been attributed to different parts of the brain - the role of the frontal lobe in executive function, the hippocampus in memory, the occipital lobe in visual processing and the parietal lobe in attention and spatial orientation have been well documented. Cognition is affected in many psychiatric conditions, from dementia to mood and psychotic disorders. A better understanding of the neuroanatomical basis of cognition would provide a deeper appreciation of a variety of brain disorders. This would help bring diagnostic clarity and therapeutic benefits. The unravelling of the anatomical substrates of cognition would shed light on a broad range of conditions from 'conversion disorders' to organic brain syndromes and malingering. This presentation explores these neuroanatomical substrates.

\section{Chronic exposure to light reverses the effects of maternal separation on the rat prefrontal cortex \\ V Russel, ${ }^{\star} \mathrm{J}$ Dimatelis}

Department of Human Biology, Faculty of Health Sciences,

University of Cape Town, South Africa

*vivienne.russell@uct.ac.za

Background. Animals subjected to maternal separation display behavioural and endocrine disturbances, as well as structural and functional changes in the prefrontal cortex and limbic areas of the brain. Objective. To determine the effect of maternal separation and treatment with either chronic constant light exposure or escitalopram on proteins in the prefrontal cortex.

Methods. Four experimental groups of male Sprague-Dawley rats were subjected to (i) normal rearing, (ii) maternal separation $(3 \mathrm{~h}$ per day from postnatal day 2 (P2) to P14), (iii) maternal separation followed by chronic light exposure (P42 - P63) or (iv) maternal separation followed by treatment with the antidepressant drug escitalopram (P68 - P100). Groups 1 - 3 were treated with saline as vehicle control for the escitalopram-treated group. At P101, all rats were decapitated and the prefrontal cortex was collected and stored at $-80^{\circ} \mathrm{C}$. Tissue from 3 rats per group was pooled and proteins determined by isobaric tagging for relative and absolute quantification (iTRAQ) using matrix-assisted laser desorption/ionisation tandem mass spectrometry (MALDI-MS/MS).

Results. Maternal separation led to a hypometabolic state in the prefrontal cortex by decreasing energy-related proteins (creatine kinase B, aconitate hydratase). Cell signalling proteins (synapsin I, calmodulin, 14-3-3 protein epsilon) and cytoskeletal proteins (spectrin, microtubule-associated protein) were reduced in the prefrontal cortex by maternal separation. Maternal separation also increased dihydropyrimidinase-related protein/collapsin response mediator protein (CRMP) and myelin proteolipid protein. CRMP impairs plasticity and neuronal signalling. Exposure of maternally separated animals to constant light during adolescence reversed the hypometabolic state by increasing energy-related proteins in the prefrontal cortex, increasing cell signalling and cytoskeletal proteins, and decreasing the expression of CRMP. Both light and escitalopram increased ATP synthase in maternally separated rats. Escitalopram also increased 2',3'-cyclic-nucleotide 3'-phosphodiesterase (CNPase) and myelin proteolipid protein in maternally separated rats. 
Conclusion. Constant light exposure during adolescence reversed a range of protein changes in prefrontal cortex of rats exposed to early maternal separation. In particular, the increased expression of CRMP by maternal separation was reversed by light treatment. The effects of light exposure overlapped partially with the effects of escitalopram treatment.

\section{Evaluating a new drug to combat Alzheimer's disease S Sibiya, ${ }^{*}$, W M U Daniels, M V Mabandla \\ Discipline of Human Physiology, School of Laboratory Medicine and Medical Sciences, University of KwaZulu-Natal, Durban, South Africa *208510719@stu.ukzn.ac.za}

Background. Alzheimer's disease (AD) is the most prevalent form of dementia affecting the elderly population, affecting $10 \%$ of the population $>65$ years of age and $50 \%$ of the population $>85$ years of age. Hallmark lesions include, extracellular deposition of $\beta$-amyloid protein fibrillar plaques $(A \beta)$ and intraneuronal neurofibrillary tangles (NFTs), which aggregate and impair synaptic plasticity in the target regions of the brain, causing a progressive decline in cognitive functioning. Current therapies of $\mathrm{AD}$ are merely palliative and only slow down cognitive decline. Treatments that address underlying pathologic mechanisms are completely lacking. In a recent study a novel compound poly-N-methylated amyloid Beta (A $\beta$ )-peptide $\mathrm{C}$-Terminal Fragments (MEPTIDES) has been shown to reduce A $\beta$ toxicity in vitro and in Drosophila melanogaster. However it is unclear whether this novel drug is equally effective in mammals.

Objective. To investigate the effects MEPTIDES, in the reduction of neurotoxicity of $\beta$-Amyloid on the rat brain, thus suggesting it as a possible AD treatment drug.

Methods. Fully grown male Sprague-Dawley rats $(n=66)$, between 300 and $350 \mathrm{~g}$ (8/9 weeks of age) will be used in this study. The study will be divided into 2 parts; first 30 rats will be used to establish a plausible concentration/dose dependent curve for $\mathrm{A} \beta$ that produces the greatest cognitive impairment, hence establishing a plausible model. Second, the remainder will be randomly assigned into 2 control groups and 2 experimental groups ( $\mathrm{A} \beta$ and saline). All groups will receive an intracerebral injection of either $A \beta$ or saline. Learning and memory tests will be performed using the Morris water maze. The drug or saline will be administered at a point of greatest cognitive impairment in the learning and memory tasks. Brain and hippocampal tissue will be analysed by polymerase chain reaction (PCR), Western Blot, Matrix-assisted laser desorption/ionisation times of flight MALDI-TOF and histology.

Results. We hope to show improved learning and memory in animals treated with the drug MEPTIDES, suggesting it as a potential management therapy/treatment for $\mathrm{AD}$ progression. This will have substantial medical, social and financial benefit for patients, family members and the healthcare fraternity.

\section{Structural brain changes in HIV-infected women with and without childhood trauma \\ G Spies, ${ }^{1 \star}$ F Ahmed, ${ }^{1}$ C Fennema-Notestine, ${ }^{3,4}$ S Archibald, ${ }^{4}$ S Seedat $^{1,2}$ \\ ${ }^{1}$ South African Research Chairs Initiative (SARChI), PTSD program, Department of Psychiatry, Stellenbosch University, South Africa;}

${ }^{2}$ MRC Unit on Anxiety and Stress Disorders, Department of Psychiatry, Stellenbosch University, South Africa;

${ }^{3}$ Department of Psychiatry, University of California San Diego, La Jolla, California, USA

${ }^{4}$ Department of Radiology, University of California San Diego, La Jolla, California, USA

*ggiocos@sun.ac.za

Background. HIV-infected patients commonly have neurocognitive deficits. HIV is also associated with morphological brain abnormalities affecting neural substrates that subserve neurocognitive function. Early life stress (ELS) can also directly influence brain morphology. Objective. Our hypothesis was twofold: firstly that women with HIV and ELS would have significantly reduced regional brain volumes (hippocampus, corpus callosum, anterior cingulate) compared with HIV-positive women without ELS; and secondly, that HIV-positive women would have significantly reduced brain volumes compared with HIV negative women, which would be consistent with greater neurocognitive impairments.

Methods. Structural data were captured using a 3T Magnetom MRI scanner. We used Freesurfer to determine between-group volumetric differences and examined the relationship between cognition and brain volumes. A battery of neurocognitive tests was administered to 67 matched women: HIV-positive with ELS ( $n=26)$, HIV-positive without ELS ( $n=17)$, HIV-negative with and without ELS $(n=24)$, mean age 29.34 years.

Results. Analysis of covariance (ANCOVA) revealed no significant main effects for regions of interest across the 3 groups. Multivariate linear regression analyses between neurocognition and HIV-positive women with and without ELS revealed significant associations in the hippocampus bilaterally for executive functions and working memory, while left anterior cingulate cortex (ACC) was significantly associated with executive functions and learning and the corpus callosum was significantly associated with executive functions. Multivariate linear regression analyses of the association of neurocognition and brain volumes in HIV-positive compared with HIV-negative women showed that speed of information processing, working memory, and executive functions were significantly associated with bilateral hippocampal reductions. Reduced volume of the right ACC was significantly associated with learning deficits and reduced corpus callosal volume was significantly associated with impairments in executive function.

Conclusion. This appears to be the first documented volumetric and neurocognitive study in HIV-infected individuals with ELS. Our results demonstrated differential interaction effects for trauma and HIV regarding neurocognitive deficits and regional volumetric findings.

\section{Nicotine-stimulated release of hippocampal norepinephrine is reduced in an animal model of attention-deficit/ hyperactivity disorder: the spontaneously hypertensive rat T Sterley \\ Department of Human Biology, University of Cape Town, South Africa tsterley@gmail.com}

Background. Attention-deficit/hyperactivity disorder (ADHD) is a heterogeneous developmental disorder and is one of the most 
common child psychiatric disorders. It is also a risk factor for early smoking and adult nicotine dependence. Nicotine has been shown to improve symptoms associated with $\mathrm{ADHD}$, including attention, working memory and response inhibition. Norepinephrine is a neurotransmitter involved in attention. It is highly implicated in ADHD, and often targeted in treatment.

Objective. We investigated nicotine's effect on release of norepinephrine in the hippocampus of a validated rat model of ADHD, the spontaneously hypertensive rat (SHR), and in 2control strains: WistarKyoto rats (WKY) and Sprague-Dawley rats (SD).

Methods. Hippocampal slices from male SHR, WKY and SD (postnatal day 31 - 33) were prelabelled with radioactively labelled norepinephrine $\left(\left[{ }^{3} \mathrm{H}\right] \mathrm{NE}\right)$ and perfused with buffer. The slices were stimulated by exposure to different concentrations of nicotine $(1,10,100$ or 1000 $\mu \mathrm{M})$ for 1 minute at two intervals ( $\mathrm{S} 1$ and $\mathrm{S} 2$, separated by 20 minutes). Since glutamate and Gamma-Aminobutyric acid (GABA) receptor function differ in SHR compared with WKY, we also investigated the involvement of glutamatergic 2-amino-3-(3-hydroxy-5-methylisoxazol-4-yl)propanoic acid (AMPA) receptors and $\mathrm{GABA}_{\mathrm{A}}$ receptors in nicotine $(100 \mu \mathrm{M})$-stimulated release of hippocampal $\left[{ }^{3} \mathrm{H}\right] \mathrm{NE}$ in each of the strains by blocking these receptors with 6-cyano-7nitroquinoxaline-2,3-dione (CNQX) (AMPA receptor antagonist, 10 $\mu \mathrm{M})$ or bicuculline $\left(\mathrm{GABA}_{\mathrm{A}}\right.$ receptor antagonist, $\left.30 \mu \mathrm{M}\right)$ respectively.

Results. Nicotine-stimulated release (S1) of $\left[{ }^{3} \mathrm{H}\right] \mathrm{NE}$ from SHR hippocampal slices was less than that of WKY and SD, at $100 \mu \mathrm{M}$ and 1 $000 \mu \mathrm{M}$ nicotine. This may suggest reduced density and/or function of nicotinic receptors on noradrenergic varicosities in SHR hippocampus. Nicotine-stimulated release of $\left[{ }^{3} \mathrm{H}\right] \mathrm{NE}$ in response to $\mathrm{S} 2$ was reduced compared to $\mathrm{S} 1$ in all strains, possibly indicating desensitization of the nicotine NE-stimulation pathway. Blocking AMPA receptors with CNQX had no effect on nicotine-stimulated release of $\left[{ }^{3} \mathrm{H}\right] \mathrm{NE}$ in any of the strains. In WKY, nicotine-stimulated release of $\left[{ }^{3} \mathrm{H}\right] \mathrm{NE}$ was reduced by $\mathrm{GABA}_{\mathrm{A}}$ receptor antagonist, bicuculline.

Conclusion. Nicotine-stimulated release of $\left[{ }^{3} \mathrm{H}\right] \mathrm{NE}$ from SHR is reduced compared to that of WKY and $\mathrm{SD}$, and also lacks the $\mathrm{GABA}_{\mathrm{A}}$ receptor component that is evident in WKY. These neurochemical differences between SHR and WKY may underlie their very different behaviours and learning abilities. We conclude that reduced nicotinic receptor activity, and reduced involvement of GABAA receptors in nicotine receptor activity, may be implicated in ADHD neuropathology.

\section{Brain-derived neurotrophic factor (BDNF) protein levels in anxiety disorders: Systematic review and meta-regression analysis \\ $\underline{\text { S Suliman }},{ }^{1 *}$ S M J Hemmings, ${ }^{2,3}$ S Seedat ${ }^{1,3}$ \\ ${ }^{1}$ MRC Anxiety Disorders Unit, Department of Psychiatry, Faculty of Medicine and Health Sciences, Stellenbosch University, South Africa \\ ${ }^{2}$ Division of Molecular Biology and Human Genetics, Faculty of \\ Medicine and Health Sciences, Stellenbosch University, South Africa \\ ${ }^{3}$ Department of Psychiatry, Faculty of Medicine and Health Sciences, \\ University of Stellenbosch, South Africa \\ *sharain@sun.ac.za}

Background. Brain-derived neurotrophic factor (BDNF) is a neurotrophin that is involved in the synaptic plasticity and survival of neurons. BDNF is believed to be involved in the pathogenesis of several neuropsychiatric disorders.

Objective. As findings of BDNF levels in the anxiety disorders have been inconsistent, we conducted a systematic review and meta-analysis of studies that assessed BDNF protein levels in anxiety disorders.

Methods. We conducted the review using electronic databases and searched reference lists of relevant articles for any further studies. Studies that measured BDNF protein levels in any anxiety disorder and compared these with a control group were included. Effect sizes of the differences in BDNF levels between anxiety disorder and control groups were calculated.

Results. Eight studies with a total of 1179 participants were included. BDNF levels were found to be lower in individuals with any anxiety disorder compared to those without (mean -0.94 , range -1.75 to -0.12 ; $p \leq 0.05)$. This finding differed with regards to source of BDNF protein (plasma mean -1.31; range -1.69 to $-0.92, p \leq 0.01$; serum: mean -1.06 , range -2.27 to $0.16, p \geq 0.01$ ) and type of anxiety disorder (post-traumatic stress disorder mean -0.05 , range $-1.66 ; 1.75, p \geq 0.01$; obsessive compulsive disorder mean -2.33 , range -4.21 to $-0.45 ; p \leq 0.01$ ).

Conclusion. Although BDNF levels are reduced in individuals with an anxiety disorder, this is not consistent across the various anxiety disorders and appears to be mediated by differences in sampling methods. Findings are, however, limited by the lack of research in this area, and given the potential for BDNF as a biomarker of anxiety disorders it would be useful to clarify the relationship further.

\section{A 12-month retrospective audit of the demographic and clinical profile of mental healthcare users admitted to a district level hospital in the Western Cape, South Africa E Thomas, ${ }^{1 \star}$ K J Cloete, ${ }^{1}$ M Kidd, ${ }^{2}$ H Lategan ${ }^{1}$ \\ ${ }^{1}$ Department of Psychiatry, Faculty of Medicine and Health Sciences, Stellenbosch University, Cape Town, South Africa \\ ${ }^{2}$ Centre for Statistical Consultation, Stellenbosch University, Cape Town, South Africa \\ *lene_tnt@live.co.za}

Background. The Western Cape Province has the highest 12-month and lifetime mental illness prevalence rates in South Africa. The Mental Health Care Act of 2002 selected regional and district public hospitals to admit, observe, and treat mental healthcare patients for 72 hours prior to psychiatric hospital admission. These hospitals face infrastructural and human resource challenges, aggravated by inadequate mental healthcare user data.

Objective. This study was a 12-month retrospective review of a typical designated 72-hour assessment unit in the Western Cape.

Methods. This review included 487 admission episodes of mental healthcare users admitted to Helderberg Hospital between 1 January 2011 and 31 December 2011. We collected data on demographic information, clinical diagnosis, length of stay (including data from those not discharged during the study period), referral pattern to the next level of psychiatric care or other clinical departments, and outcomes (absconsion, discharge, readmission, transfer, or death).

Results. Patients were predominantly male gender (55\%), with a mean \pm standard deviation age of $34 \pm 14$ years. The most prevalent diagnoses resulting from substance abuse, specifically 
methamphetamine, was psychosis. Of all the admission episodes, 219 (45\%) had comorbid/secondary diagnoses and 187 (38\%) were substance-use related, with the latter accounting for 37 (57\%) of readmissions (mostly within 3 months of discharge). A total of $31(6 \%)$ absconsion-episodes were recorded, of which 19 (61\%) were substancerelated admissions. The length of stay (mean \pm SD 9.4 \pm 5.7 days) was significantly $(p<0.01)$ longer in those transferred to a psychiatric hospital than those who were not transferred (mean \pm SD $4.7 \pm 4.5$ days). Conclusions. Substance abuse, specifically methamphetamine, places a significant burden on the provision of mental healthcare. Only a small proportion of admissions were referred to specialist/tertiary mental hospitals. Adequate seclusion rooms must be provided to optimise psychiatric care, treatment, and rehabilitation as stipulated in the Mental Health Care Act of 2002. An integrative comprehensive substance abuse intervention system must be implemented.

Magnesium recurarization: A comparison between reversal of neuromuscular block with sugammadex v. neostigmine/ glycopyrrolate in an in vivo rat model

M van den Berg, ${ }^{1 \star}$ M F M James, ${ }^{2}$ L A Kellaway ${ }^{1}$

${ }^{1}$ Department of Human Biology, University of Cape Town, South Africa

${ }^{2}$ Department of Anaesthesia, University of Cape Town, South Africa

*vbrmau001@myuct.ac.za

Background. Recurarisation and residual neuromuscular block in the post-anaesthesia care unit have been linked to postoperative respiratory complications and mortality. Intravenous magnesium sulphate has been known to cause recurarisation when administered postoperatively after neuromuscular block reversal with neostigmine. Objective. Differences in magnesium recurarisation effects were compared between sugammadex and neostigmine/glycopyrrolate combination for the reversal of rocuronium neuromuscular block.

Methods. An in vivo rat sciatic nerve-gastrocnemius muscle model was used, measuring the fade of response to low frequency train-of-four (TOF) stimulation and twitch height as measures of neuromuscular transmission. Neuromuscular block was induced with rocuronium $(0.5 \mathrm{mg} / \mathrm{kg})$. Neuromuscular block was reversed upon spontaneous recovery to a TOF-ratio of 0.2 with either sugammadex (2 mg/kg-1) or neostigmine/glycopyrrolate $(0.04 \mathrm{mg} / \mathrm{kg} / 0.001 \mathrm{mg} /$ $\mathrm{kg})$ combination. Magnesium sulphate $(30 \mathrm{mg} / \mathrm{kg})$ was administered at recovery to approximately a TOF-ratio of 0.9 , and the maximum decrease in TOF-ratio and twitch height was measured.

Results. Recovery of time of flight (TOF) ratio was significantly faster with reversal using sugammadex compared with neostigmine as measured by TOF ratio; 0.5 (72s v. 255 s), 0.6 (90s v. 366.6s) and 0.7 (111.7s v. 521.3 s) $(p<0.0001)$. Recovery of twitch height was also significantly faster with sugammadex reversal compared to neostigmine, as measured by \%T1baseline; $30 \%$ (30.1 s v. 176.3 s), 40\% (42.2 s v. 201.7 s), 50\% (47.5 s v. 266.4 s), $60 \%$ (77.3 s v. 356.2 s), $70 \%$ (97.2 s v. 515.8 s) and $80 \%$ (145.6 s v. $808.7 \mathrm{~s})(p<0.05)$. The occurrence of magnesium recurarisation, as measured by decreases in the TOF-ratio $(0.33 \mathrm{v} .0 .34)$ and twitch height ( $24.9 \%$ vs. $20.2 \%$ ) was observed in both sugammadex and neostigmine/ glycopyrrolate groups, with no significant differences between the groups. Conclusion. The accelerated reversal of neuromuscular block with sugammadex compared with neostigmine/glycopyrrolate confirms previous findings. However the occurrence of magnesium recurarisation after recovery from neuromuscular block could not be prevented when sugammadex reversal was used compared with neostigmine/glycopyrrolate reversal. The safety of intravenous magnesium sulphate administration after recovery from neuromuscular block can therefore not be assured, irrespective of neuromuscular block reversal regime.

\section{Identification of breast cancer patients at increased risk of 'chemobrain': Case study and review of the literature N van der Merwe, ${ }^{1 \star} \mathrm{R}$ Pienaar, ${ }^{2}$ S J van Rensburg, ${ }^{1} \mathrm{~J}$ Bezuidenhout, ${ }^{1}$ M J Kotze ${ }^{1}$ \\ ${ }^{1}$ Department of Pathology, Faculty of Health Sciences, \\ Stellenbosch University, Cape Town, South Africa \\ ${ }^{2}$ Oncology Unit, Panorama Medi-Clinic, Cape Town, South Africa \\ *colahbear@gmail.com}

Background. Cognitive decline in breast cancer patients receiving chemo- and/or endocrine therapy is becoming an issue of great clinical importance. Efforts to determine the underlying causes of comorbid neurocognitive disorders supports a shift towards more holistic approaches to breast cancer management. Variation in clinically relevant genes underlying shared disease pathways may explain the link between the metabolic syndrome and development of comorbidities such as cardiovascular disease, dementia/Alzheimer's disease, depression and adverse treatment responses. These observations supported the development of a pathology-supported genetic testing (PSGT) service, that is linked to a health outcomes research project.

Objective. We report our initial findings in relation to current evidence in the literature regarding cognitive implications in breast cancer survivors.

Methods. Having screened the first 50 breast cancer patients using a gene panel including functional single nucleotide polymorphisms (SNPs) in the apolipoprotein E (ApoE), catechol-o-methyltransferase (COMT), manganese superoxide dismutase (MnSOD) and methylenetetrahydrofolate reductase (MTHFR) genes, we performed an extensive literature review for comparative analyses.

Results and case study. Various studies suggested that recipients of chemotherapy plus tamoxifen displayed the broadest cognitive decline, with changes in visual memory and verbal working memory, whereas women treated solely with chemotherapy displayed declines mostly in working memory. In accordance with previous findings, we observed rapid development of chemobrain in a 52-year-old breast cancer patient shortly after receiving chemotherapy. This could be ascribed to detection of the ApoE E4 allele, known to be associated with both cardiovascular and Alzheimer's disease. Raised cholesterol levels, as well as several other deleterious genetic variations, were evidence of cumulative risk in this patient.

Conclusion. Data gathered from the literature and the case study underscores the importance of a multidisciplinary approach to elucidating the mechanisms underlying treatment-related toxicity in breast cancer survivors. Dissection of pharmacogenetic, lifestyle and environmental contributions to cancer co-morbidities might facilitate an improved genetic service for South African patients with breast cancer. 
The protective role of HAART and NACA in HIV Tat protein-induced hippocampal cell death

$\underline{\text { S Zulu, }}{ }^{*}$ W M U Daniels, M V Mabandla

School of Laboratory Medicine and Medical Science, College of Health

Science, University of KwaZulu-Natal, Durban, South Africa

*207506590@ukzn.ac.za

Background. HIV-induced cognitive impairment is among the most common neurological disorders associated with AIDS. Despite the introduction of highly active antiretroviral therapy (HAART), continuous neuronal cell death remains a problem for AIDS patients. The development of HIV-associated dementia is mainly due to oxidative stress. Studies have shown that transregulatory protein (Tat) induces oxidative stress in neurons, suggesting that HAART alone is not enough in addressing the complex nature of HIV and an alternative or supplementary therapy is needed. However, there is limited literature on the role of antiretroviral drugs on Tat-induced neurotoxicity.

Objective. To investigate $(i)$ the role of HAART on Tat-induced oxidative stress in hippocampal neurons and (ii) whether
$\mathrm{N}$-acetylcysteine amide (NACA) can alleviate the effects of Tatinduced neurotoxicity.

Methods. Sprague-Dawley rats $(n=60)$ with a weight of $250-300 \mathrm{~g}$ will be randomly divided into 6 groups of 10: (1) Tat (2) Tat + NACA (3) Tat + HAART (4) Saline (5) saline + HAART (6) saline + NACA. Tat (5 $\mu \mathrm{g} / 10 \mu \mathrm{l})$ will be injected bilaterally into the dorsal hippocampus using stereotaxic techniques. Some animals will then receive an intraperitoneal (i.p) injection of NACA $(250 \mathrm{mg} / \mathrm{kg}$ body weight) or saline $(10 \mu \mathrm{l})$ while HAART $(10.5 \mathrm{mg} / \mathrm{kg}$ ) will be administered by oral gavage. After treatments, hippocampal tissue will be collected for neurochemical analysis. Measuring of caspase-3 as a mark for apoptosis has been done on flow cytometry. Western blots will be used to detect protein carbonyl and 4-hydroxynonenal, immunohistology of glial fibrillary acidic protein. Enzyme-linked immunosorbent assay (ELISA) for TNF- $\alpha$ will also be performed.

Results. Flow cytometry detection of active caspase- 3 has shown a low percentage of active caspase- 3 on Tat + saline animals.

Conclusion. Tat protein does not cause neuron apoptosis. This suggests that Tat may cause neuronal dysfunction rather than neuronal death. 